\title{
MOVABLE CURVES AND SEMISTABLE SHEAVES
}

\author{
DANIEL GREB, STEFAN KEBEKUS, AND THOMAS PETERNELL
}

\begin{abstract}
This paper extends a number of known results on slope-semistable sheaves from the classical case to the setting where polarisations are given by movable curve classes. As applications, we obtain new flatness results for reflexive sheaves on singular varieties, as well as a characterisation of finite quotients of Abelian varieties via a Chern class condition.
\end{abstract}

\section{CONTENTS}

1. Introduction 1

2. Semistability with respect to a movable class 3

3. Openness of semistability 8

4. Tensor products of semistable sheaves 11

5. Bogomolov-Gieseker inequalities 14

6. Flatness criteria 17

7. Characterisation of torus quotients 21

References 23

\section{INTRODUCTION}

Given an $n$-dimensional, complex, projective manifold $X$, the notion of stability is arguably the single most important concept in the discussion of sheaves of $\mathscr{O}_{X^{-}}$ modules and their moduli. A widely-used stability notion is slope-stability. Its wellknown definition depends on the choice of a very ample line bundle $\mathscr{H} \in \operatorname{Pic}(X)$ and asks to associate to any sheaf $\mathscr{E}$ of positive rank the slope

$$
\mu_{\mathscr{H}}(\mathscr{E}):=\frac{c_{1}(\mathscr{H})^{n-1} \cdot c_{1}(\operatorname{det} E)}{\operatorname{rank} \mathscr{E}}=\frac{[C] \cdot c_{1}(\operatorname{det} E)}{\operatorname{rank} \mathscr{E}},
$$

where $[C]$ is the numerical class of a curve $C$, obtained as the intersection of general elements $\left(H_{i}\right)_{1 \leq i<n} \in|\mathscr{H}|$, that is, $C:=H_{1} \cap \cdots \cap H_{n-1}$. The sheaf $\mathscr{E}$ is said to be semistable with respect to $\mathscr{H}$ if $\mathscr{E}$ is torsion free and if $\mu_{\mathscr{C}}(\mathscr{F}) \leq \mu_{\mathscr{H}}(\mathscr{E})$ for any non-zero subsheaf $\mathscr{F} \subseteq \mathscr{E}$.

It turns out that these notions are often not flexible enough to allow for applications in higher-dimensional birational geometry. There, one frequently needs to discuss a number of birational models, and compare sheaves that live on one model with sheaves that live on another. However, the pull-back of an ample polarisation is generally not ample. In this setting, it is often advantageous to generalise the notion of slope, replacing the class $[C]$ with a general movable curve class. Recall that a numerical curve class $\alpha$ is movable if $D \cdot \alpha \geq 0$ for all effective divisors

Date: 16th April 2015.

All three authors were partially supported by the DFG-Forschergruppe 790 "Classification of Algebraic Surfaces and Compact Complex Manifolds". Stefan Kebekus acknowledges additional support through a joint fellowship of the Freiburg Institute of Advanced Studies (FRIAS) and the University of Strasbourg Institute for Advanced Study (USIAS). 
D. Sample applications in birational geometry are given in [Miy87, CP11, GKP14]. In a different direction, the paper [GT13] uses polarisations by movable curves to resolve pathological wall-crossing phenomena for moduli spaces of sheaves on higher-dimensional varieties.

The present paper extends a number or known results from the classical case to the setting where polarisations are given by movable curve classes. As applications, we prove new flatness results for reflexive sheaves on singular varieties, as well as a uniformisation result.

1.1. Outline of the paper. We begin in Section 2 with a brief account of the relevant definitions and of elementary properties, including the existence of Harder-Narasimhan and Jordan-Hölder filtrations, boundedness results and a weak Mehta-Ramanathan theorem. Once these basics are established, the following aspects will be discussed in the remainder of the paper.

1.1.1. Openness of stability. We will show in Section 3 that stability is an open property in the interior of the movable cone. Somewhat more generally, we show that if a sheaf $\mathscr{E}$ is stable with respect to a movable class $\alpha$ that lies on the boundary of the movable cone, then $\alpha$ can be approximated by a sequence of $\mathscr{E}$-stabilising classes from the interior.

1.1.2. Semistability of tensor products. When semistability is defined with respect to an ample class, a classical theorem (specific to characteristic zero) asserts that the tensor product of two semistable locally free sheaves is again semistable, [HL10, Thm. 3.1.4]. Theorem 4.2 of Section 4 extends this result to sheaves that are semistable with respect to movable curve classes on possibly singular spaces.

Remark 1.1 (Erratum to [GKP14]). The assertion of Theorem 4.2 is stated in [GKP14] as "Fact A.13", referring to [CP11, Thm. 5.1 and Cor. 5.2] for a proof. However, these references work under the additional assumption that the movable class $\alpha \in N_{1}(X)_{\mathbb{Q}}$ is big, that is, contained in the interior of the movable cone. This assumption is not necessarily satisfied in the setting of [GKP14]. Theorem 4.2 fills this gap.

1.1.3. Bogomolov-Gieseker inequalities. Section 5 discusses the Bogomolov-Gieseker inequality for movable polarisations on a smooth surface: if $\mathscr{E}$ is a torsion-free coherent sheaf of rank $r$ on a smooth projective surface, which is semistable with respect to some non-zero movable curve class, then there is an inequality of Chern numbers, $2 r \cdot c_{2}(\mathscr{E}) \geq(r-1) \cdot c_{1}^{2}(\mathscr{E})$.

1.1.4. Flatness criteria. The Bogomolov-Gieseker inequality will be applied in Section 6 to generalise a flatness criterion of Simpson. Let $X$ be smooth, complex, projective surface $X$, let $\alpha$ be a movable class and $\mathscr{E}$ be a torsion-free sheaf such that the following numbers vanish,

$$
c_{1}(\mathscr{E}) \cdot \alpha=c_{1}(\mathscr{E})^{2}-c_{2}(\mathscr{E})=0 .
$$

If $\mathscr{E}$ is $\alpha$-semistable and $\alpha^{2}>0$, then $\mathscr{E}$ is a locally free, flat sheaf. In other words, $\mathscr{E}$ is given by a linear representation of the fundamental group. We also obtain a criterion for projective flatness, Theorem 6.4.

The following theorem is a consequence of the flatness criterion and of results obtained by the authors in [GKP13]. For convenience, the following notation is used. If $f: A \rightarrow B$ is any morphism of quasi-projective varieties and if $\mathscr{S}$ is any coherent sheaf of $\mathscr{O}_{B}$-modules, write $f^{[*]} \mathscr{S}:=\left(f^{*} \mathscr{S}\right)^{* *}$. A finite, surjective morphism is called quasi-étale if it is unbranched in codimension one. 
Theorem 1.2 (Flatness criterion $=$ Theorem 6.5 on page 17). Let $X$ be a normal, projective, Q-factorial variety of dimension $n$ with only canonical singularities. Let $\mathscr{E}$ be a reflexive sheaf on $X$ and $H \in \operatorname{Div}(X)$ an ample divisor. Suppose that $\mathscr{E}$ is $H$-semistable and that there exists a desingularisation $\pi: \widetilde{X} \rightarrow X$ such that the following two equalities hold,

$$
0=c_{1}(\mathscr{E}) \cdot H^{n-1} \quad \text { and } \quad 0=c_{1}\left(\pi^{[*]} \mathscr{E}\right)^{2} \cdot\left(\pi^{*} H\right)^{n-2}-c_{2}\left(\pi^{[*]} \mathscr{E}\right) \cdot\left(\pi^{*} H\right)^{n-2} .
$$

Then, there exists a quasi-étale morphism $\gamma: \widehat{X} \rightarrow X$ such that $\gamma^{[*]} \mathscr{E}$ is a locally free, flat sheaf on $\widehat{X}$.

Remark 1.3 (Coefficients in flatness criterion). In the setting of Theorem 1.2, the Hodge index theorem implies that $c_{1}\left(\pi^{[*]} \mathscr{E}\right)^{2} \cdot\left(\pi^{*} H\right)^{n-2} \leq 0$. Using the Bogomolov-Gieseker Inequality of Section 1.1.3, one obtains that the following conditions, which seemingly depend on the choice of a real parameter $\lambda$,

$$
0=c_{1}\left(\pi^{[*]} \mathscr{E}\right)^{2} \cdot\left(\pi^{*} H\right)^{n-2}-\lambda \cdot c_{2}\left(\pi^{[*]} \mathscr{E}\right) \cdot\left(\pi^{*} H\right)^{n-2},
$$

are in fact equivalent for all $0<\lambda<\frac{2 r}{r-1}$. The formulation of Theorem 1.2 uses $\lambda=1$. The choice $\lambda=2$ is often seen in the literature since it appears in the second Chern character, cf. [Sim92, Cor. 3.10].

1.1.5. Characterisation of torus quotients. In the last section we apply the flatness criteria to the cotangent sheaf of a manifold, in order to obtain the following characterisation result.

Theorem 1.4 (Characterisation of torus quotients $=$ Theorem 7.1 on page 21). Let $X$ be a normal $\mathbb{Q}$-factorial projective variety of dimension $n$ with only canonical singularities and numerically trivial canonical bundle, $K_{X} \equiv 0$. Assume that there exists a desingularisation $\pi: \widetilde{X} \rightarrow X$ and an ample divisor $H \in \operatorname{Dix}(X)$ such that $c_{2}(\widetilde{X}) \cdot\left(\pi^{*} H\right)^{n-2}=0$. Then, $X$ is smooth in codimension two, there exists an Abelian variety $A$ and a quasi-étale morphism $\gamma: A \rightarrow X$.

This result generalises a result for three-dimensional varieties of ShepherdBarron and Wilson [SBW94, Cor. of Main Thm], and eliminates the a priori assumption on the codimension of the singular locus made in [GKP13, Thm. 1.16]. After the proof of this result was completed, we learned that Lu-Taji obtained similar results, to appear in a forthcoming preprint.

1.2. Global assumptions. Throughout the paper, we work over the complex number field. If not mentioned otherwise, all sheaves are assumed to be coherent.

1.3. Acknowledgements. We would like to thank Matei Toma for important suggestions. The main idea of Theorem 3.4 goes back to him. The authors would also like to thank two anonymous referees for their helpful and very detailed reports. A part of this work was done during a very pleasant stay of the third named author at the Freiburg Institute for Advanced Studies.

\section{Semistability With RESPECT TO A MOVABLE CLASS}

2.1. Numerical classes. Given a normal projective variety $X$, we consider the space $N_{1}(X)_{\mathbb{R}}$ of numerical curve classes, as well as the space $N^{1}(X)_{\mathbb{R}}$ of numerical Cartier divisor classes. We refer the reader to [Kol96, Sect. II.4] for a brief definition and discussion of these spaces. Recall from [Kol96, Sect. II.4, (4.2.5)] that the 
intersection number of curves and Cartier divisors gives rise to a nondegenerate bilinear pairing

$$
N_{1}(X)_{\mathbb{R}} \times N^{1}(X)_{\mathbb{R}} \rightarrow \mathbb{R} .
$$

Definition 2.2 (Cone of movable curve classes). A class $\alpha \in N_{1}(X)_{\mathbb{R}}$ is called movable if $\alpha \cdot D \geq 0$ for any effective Cartier divisor $D$. The set of movable classes forms a closed, convex cone $\operatorname{Mov}(X) \subset N_{1}(X)_{\mathbb{R}}$, called the movable cone.

Remark 2.3. If $X$ is a manifold, it has been shown in [BDPP13] that the movable cone is the closure of the convex cone in $N_{1}(X)_{\mathbb{R}}$ generated by curves whose deformations cover a dense subset of $X$. A divisor class $\Delta \in N^{1}(X)_{\mathbb{R}}$ is pseudoeffective if and only if the associated function $\bullet \cdot \Delta$ is non-negative on $\operatorname{Mov}(X)$.

Definition 2.4 (Big movable class). Let $\alpha \in N_{1}(X)_{\mathbb{R}}$ be a movable class. We say that $\alpha$ is big if it lies in the interior of the movable cone.

2.1.1. Q-Cartier divisors on singular spaces. If $X$ is $\mathbb{Q}$-factorial, we briefly show that there exists a number $m$ such that for any Weil divisor $D$, the numerical class $[m$. $D]$ is the numerical class of a Cartier divisor. The following notation will be used.

Definition 2.5 (Q-Cartier divisors that are numerically Cartier). Let X be a normal, projective variety. If $D$ is any $\mathbb{Q}$-Cartier, $\mathbb{Q}-$ Weil divisor on $X$, then $D$ defines a numerical class $[D] \in N^{1}(X)_{\mathbb{Q}} \subset N^{1}(X)_{\mathbb{R}}$. We say that " $D$ is numerically Cartier" if there exists a Cartier divisor $\Delta$ whose numerical class equals that of $D$, that is, $[D]=[\Delta]$.

Definition 2.6 (Numerical classes of sheaves). Let $X$ be a normal, projective variety. If $\mathscr{F}$ is any coherent sheaf on $X$, its determinant is a Weil divisorial sheaf, say $\operatorname{det} \mathscr{F} \cong \mathscr{O}_{X}(D)$. If $D$ is $\mathbb{Q}$-Cartier, we define the numerical class of $\mathscr{F}$ as $[\mathscr{F}]:=[D] \in$ $N^{1}(X)_{\mathbb{Q}} \subset N^{1}(X)_{\mathbb{R}}$. If $X$ is smooth, we also use the traditional notation $c_{1}(\mathscr{F})=[\mathscr{F}]$.

Theorem 2.7 (Finite generation of numerical divisor classes). Let $X$ be a normal, Q-factorial, projective variety. Then, the set

$$
N_{Q \text { Div }}(X):=\{[D] \mid D \text { an integral Weil divisor }\} \subseteq N^{1}(X)_{\mathbb{R}}
$$

is a lattice which contains the lattice spanned by numerical classes of Cartier divisors. In particular, there exists a positive integer $m \in \mathbb{Q}^{+}$such that $m \cdot D$ is numerically Cartier, for any integral Weil divisor $D$ on $X$.

Proof. It suffices to show that $N_{Q \text { Div }}(X)$ is finitely generated as a $\mathbb{Z}$-module. To this end, let $\pi: \widetilde{X} \rightarrow X$ be any resolution of singularities. Since $X$ is normal and Q-factorial, push-forward of Weil divisors induces a surjective map

$$
\pi_{*}: N^{1}(\widetilde{X})_{\mathbb{Q}} \rightarrow N^{1}(X)_{\mathbb{Q}}
$$

The Theorem of the Base of Néron-Severi, [Kol96, II Th. 4.5], asserts that $N^{1}(\widetilde{X})_{\mathbb{Z}}$ is finitely generated. Its image under $\pi_{*}$ is exactly $N_{Q \text { Div }}(X)$.

2.1.2. Push-forward and pull-back. Let $\phi: X \rightarrow Y$ be any dominant, rational map between normal, Q-factorial, projective varieties. Assume that the inverse $\phi^{-1}$ does not contract any divisors - examples are given by resolutions of singularities, more generally birational morphisms, and maps obtained by running the minimal model program. In this setting, there are well-known results concerning push-forward and pull-back. In the case of rational numerical classes, these are summarised in [GKP14]. By linearity, everything said there also holds for real classes. For the reader's convenience, we recall the most important facts.

Push-forward of Weil divisors together with the pairing (2.1.1) induces linear morphisms

$$
\phi_{*}: N^{1}(X)_{\mathbb{Q}} \rightarrow N^{1}(Y)_{\mathbb{Q}} \quad \text { as well as } \quad \phi^{*}: N_{1}(Y)_{\mathbb{Q}} \rightarrow N_{1}(X)_{\mathbb{Q}} .
$$


Since the push-forward of any effective divisor is effective, it follows immediately from Definition 2.2 that the pull-back of any movable curve class is again movable.

Proposition 2.8 (Push-forward and pull-back of sheaves). Let $\phi: X \rightarrow Y$ be any dominant, birational morphism of normal, $\mathbb{Q}$-factorial, projective varieties. Let $\alpha \in N_{1}(Y)_{\mathbb{R}}$ be any class, and $\mathscr{F}, \mathscr{G}$ be torsion-free, coherent sheaves on $X$ and $Y$, respectively. Then, the following holds.

(2.8.1) We have $[\mathscr{F}] \cdot \phi^{*} \alpha=\left[\phi_{*} \mathscr{F}\right] \cdot \alpha$.

(2.8.2) If $\mathscr{F}$ agrees with $\phi^{*} \mathscr{G}$ away from the $\phi$-exceptional set, and if $(r, a) \in \mathbb{N} \times \mathbb{Z}$ is any pair of numbers, then $\mathscr{G}$ contains a subsheaf $\mathscr{G}^{\prime}$ of rank $r$ and intersection $\left[\mathscr{G}^{\prime}\right] \cdot \alpha=a$ if and only if $\mathscr{F}$ contains a subsheaf $\mathscr{F}^{\prime}$ of rank $r$ and intersection $\left[\mathscr{F}^{\prime}\right] \cdot \phi^{*} \alpha=a$ that agrees with $\phi^{*} \mathscr{G}^{\prime}$ away from the $\phi$-exceptional set.

Remark 2.9. Using the notion of (semi)stability with respect to a movable curve class, cf. Definition 2.11 below, Proposition 2.8 asserts that $\mathscr{G}$ is $\alpha$-(semi)stable if and only if $\mathscr{F}$ is $\phi^{*} \alpha$-(semi)stable.

2.2. Slope and semistability. We will use the following terminology throughout the paper.

Definition 2.10 (Slope with respect to a movable class). Let $X$ be a normal, $Q-$ factorial, projective variety and $\alpha \in \operatorname{Mov}(X)$. If $\mathscr{E} \neq 0$ is any torsion-free, coherent sheaf on $X$, one defines the slope of $\mathscr{E}$ with respect to $\alpha$ as the real number

$$
\mu_{\alpha}(\mathscr{E}):=\frac{[\mathscr{E}] \cdot \alpha}{\operatorname{rank} \mathscr{E}}
$$

Definition 2.11 (Semistability with respect to a movable class). In the setting of Definition 2.10, we say that $\mathscr{E}$ is $\alpha$-semistable if $\mu_{\alpha}(\mathscr{F}) \leq \mu_{\alpha}(\mathscr{E})$ for any coherent subsheaf $0 \subsetneq \mathscr{F} \subseteq \mathscr{E}$.

Definition 2.12 (Stability with respect to a movable class). In the setting of Definition 2.10 , we say that $\mathscr{E}$ is $\alpha$-stable if $\mu_{\alpha}(\mathscr{F})<\mu_{\alpha}(\mathscr{E})$ for any coherent subsheaf $0 \subsetneq \mathscr{F} \subsetneq \mathscr{E}$ with rank $\mathscr{F}<\operatorname{rank} \mathscr{E}$.

2.3. Elementary properties. Essentially all elementary properties satisfied by sheaves that are stable with respect to ample polarisations also hold when stability is defined by a movable class. For the reader's convenience, we summarise those properties that will be relevant later.

Proposition 2.13 (Subbundle of equal rank). Let X be a normal, Q-factorial, projective variety and $\alpha \in \operatorname{Mov}(X)$. If $\mathscr{F} \subseteq \mathscr{E}$ are two torsion-free, coherent sheaves of equal rank, then $\mu_{\alpha}(\mathscr{F}) \leq \mu_{\alpha}(\mathscr{E})$.

Proof. There exists an effective Weil-divisor $D$ such that $\operatorname{det} \mathscr{F}$ and $\operatorname{det} \mathscr{E}$ differ only by a twist with $D$, or more precisely, $\operatorname{det} \mathscr{F} \cong\left(\mathscr{O}_{X}(-D) \otimes \operatorname{det} \mathscr{E}\right)^{* *}$. In particular, we have the following equality of numerical classes,

$$
[\mathscr{F}]=[\mathscr{E}]-[D] .
$$

Since $\alpha$ is movable, we have that $[D] \cdot \alpha \geq 0$, and the claim follows.

Corollary 2.14 (Slope of saturation). Let X be a normal, Q-factorial, projective variety and $\alpha \in \operatorname{Mov}(X)$. If $\mathscr{F} \subseteq \mathscr{E}$ are two torsion-free, coherent sheaves, and if $\mathscr{F}$ sat $\subseteq \mathscr{E}$ denotes the saturation of $\mathscr{F}$ in $\mathscr{E}$, then $\mu_{\alpha}(\mathscr{F}) \leq \mu_{\alpha}\left(\mathscr{F}_{\text {sat }}\right)$.

Corollary 2.15 (Stable sheaves are semistable). Let X be a normal, Q-factorial, projective variety and $\alpha \in \operatorname{Mov}(X)$. Then, $\alpha$-stable sheaves are $\alpha$-semistable. 
Corollary 2.16 (Semistability of direct sum of line bundles). Let $X$ be a normal, Qfactorial, projective variety. If $\mathscr{H} \in \operatorname{Pic}(X)$ is any line bundle and $r \in \mathbb{N}^{+}$any number, then $\mathscr{H}^{\oplus r}$ is semistable with respect to any movable class.

The following proposition follows from elementary Chern class computations, which we leave to the reader.

Proposition 2.17 (Morphism from a semistable sheaf). Let X be a normal, Q-factorial, projective variety and $\alpha \in \operatorname{Mov}(X)$. If $\mathscr{F}$ is any $\alpha$-semistable sheaf and $\gamma: \mathscr{F} \rightarrow \mathscr{E}$ any morphism of torsion-free $\mathscr{O}_{X}$-modules, then $\mu_{\alpha}($ Image $\gamma) \geq \mu_{\alpha}(\mathscr{F})$.

Corollary 2.18 (Morphisms between semistable sheaves). Let $X$ be a normal, $Q$ factorial, projective variety and $\alpha \in \operatorname{Mov}(X)$.

- If $\mathscr{F}$ and $\mathscr{E}$ are semistable and if $\mu_{\alpha}(\mathscr{F})>\mu_{\alpha}(\mathscr{E})$, then $\operatorname{Hom}_{\mathscr{O}_{X}}(\mathscr{F}, \mathscr{E})=0$.

- If $\mathscr{F}$ and $\mathscr{E}$ are stable and if $\mu_{\alpha}(\mathscr{F})=\mu_{\alpha}(\mathscr{E})$, then any non-zero morphism $\mathscr{F} \rightarrow \mathscr{E}$ is injective and generically isomorphic. If we assume in addition that $\mathscr{F}$ is saturated ${ }^{1}$ in $\mathscr{E}$, then any non-zero morphism $\mathscr{F} \rightarrow \mathscr{E}$ is an isomorphism.

2.4. A generalisation of Mehta-Ramanathan's theorem. This section contains a minor generalisation of the classical theorem of Mehta-Ramanathan.

Proposition 2.19. Let $\pi: \widetilde{X} \rightarrow X$ be a birational morphism of normal, Q-factorial, projective varieties of dimension $n \geq 2$ with only canonical singularities. Let $H$ be an ample divisor on $X$ and $\widetilde{H}:=\pi^{*} H$. Let $\widetilde{E}$ be a torsion-free, $\widetilde{H}$-semistable sheaf on $\widetilde{X}$. If $m \gg 0$ is sufficiently large and $\widetilde{D}_{1}, \ldots, \widetilde{D}_{n-2} \in|m \widetilde{H}|$ is a general $(n-2)$-tuple of hypersurfaces with associated complete intersection surface $\widetilde{S}=\widetilde{D}_{1} \cap \ldots \cap \widetilde{D}_{n-2}$, then $\left.\widetilde{\mathscr{E}}\right|_{\widetilde{S}}$ is $\left.\widetilde{H}\right|_{\widetilde{S}}$-semistable.

Remark 2.20. In the setting of Proposition 2.19, recall from [GKP13, Prop. 5.1] that the sheaf $\left.\widetilde{\mathscr{E}}\right|_{\widetilde{S}}$ is torsion-free. The surface $\widetilde{S}$ has canonical singularities, [KM98, Lem. 5.7], and is therefore Q-factorial, [KM98, Prop. 4.11]. The assertion that $\left.\widetilde{\mathscr{E}}\right|_{\widetilde{S}}$ is $\left.\widetilde{H}\right|_{\widetilde{S}}$-semistable therefore makes sense.

Proof of Proposition 2.19. Observe that the torsion-free coherent sheaf $\mathscr{E}:=\pi_{*}(\widetilde{\mathscr{E}})$ is $H$-semistable by Proposition 2.8. Next, write $\widetilde{D}_{i}=\pi^{*}\left(D_{i}\right)$ and set $S:=D_{1} \cap$ $\ldots D_{n-2}$ so that $\widetilde{S}=\pi^{-1}(S)$. Since $X$ has only canonical singularities, conclude as before that $S$ is $Q$-factorial. Also, observe that $\left.\mathscr{E}\right|_{S}$ is torsion-free. By Flenner's version of the Mehta-Ramanathan theorem, [Fle84, Thm. 1.2], the torsion free sheaf $\left.\mathscr{E}\right|_{S}$ is $\left.H\right|_{S}$-semistable. Since $\left.\widetilde{\mathscr{E}}\right|_{\widetilde{S}}$ and $\left(\left.\pi\right|_{\widetilde{S}}\right)^{*}\left(\left.\mathscr{E}\right|_{S}\right)$ agree outside the exceptional locus of $\left.\pi\right|_{\widetilde{S}}$, Proposition 2.8 applies, showing that $\left.\widetilde{\mathscr{E}}\right|_{\widetilde{S}}$ is $\left.\widetilde{H}\right|_{\widetilde{S}}$-semistable.

2.5. Boundedness I: Suprema of slopes in a given bundle. Given a torsion-free sheaf $\mathscr{E}$ and a movable class $\alpha$, the $\alpha$-slope of subsheaves $\mathscr{F} \subseteq \mathscr{E}$ cannot be arbitrarily large. The following boundedness results will be used later.

Definition 2.21 (Suprema of slopes). Let X be a normal, $Q$-factorial, projective variety and $\alpha \in \operatorname{Mov}(X)$. If $\mathscr{E}$ is any torsion-free, coherent sheaf of positive rank on $X$, write

$$
\mu_{\alpha}^{\max }(\mathscr{E}):=\sup \left\{\mu_{\alpha}(\mathscr{F}) \mid 0 \neq \mathscr{F} \subseteq \mathscr{E} \text { a coherent subsheaf }\right\} .
$$

Proposition 2.22 (Boundedness of $\mu_{\alpha}^{\max }$ ). In the setting of Definition 2.21, the function

$$
m: \operatorname{Mov}(X) \rightarrow \mathbb{R} \cup\{\infty\}, \quad \beta \mapsto \mu_{\beta}^{\max }(\mathscr{E})
$$

is bounded from above by a linear function $M$. In particular, $\mu_{\alpha}^{\max }(\mathscr{E})<\infty$.

\footnotetext{
${ }^{1}$ Recall that $\mathscr{F}$ is said to be saturated in $\mathscr{E}$ if the quotient $\mathscr{E} / \mathscr{F}$ is torsion-free.
} 
Proof. Choose a sufficiently ample line bundle $\mathscr{H}$ and an embedding $\mathscr{E} \hookrightarrow \mathscr{H} \oplus$, where $r:=\operatorname{rank} \mathscr{E}$. If $\mathscr{F} \subseteq \mathscr{E}$ is any coherent subsheaf, it follows from Corollary 2.16 that $\mu_{\alpha}(\mathscr{F}) \leq \mu_{\alpha}\left(\mathscr{H}^{\oplus r}\right)=[\mathscr{H}] \cdot \alpha$ for every $\alpha \in \operatorname{Mov}(X)$. We can therefore take $M(\beta):=[\mathscr{H}] \cdot \beta$ as the desired linear function.

Proposition 2.23 (Existence of subsheaves with maximal slope, I). In the setting of Definition 2.21, the supremum $\mu_{\alpha}^{\max }$ is a maximum. In other words, there exists a nonzero, coherent subsheaf $\mathscr{F} \subseteq \mathscr{E}$ such that $\mu_{\alpha}^{\max }(\mathscr{E})=\mu_{\alpha}(\mathscr{F})<\infty$.

Proof. Argue by contradiction, and assume that $\mu_{\alpha}(\mathscr{F})<\mu_{\alpha}^{\max }(\mathscr{E})$ for any nonzero, coherent subsheaf $\mathscr{F} \subseteq \mathscr{E}$. One can then find a sequence of subsheaves $\left(\mathscr{F}_{i}\right)_{i \in \mathbb{N}}$ such that the following holds.

(2.23.1) The sequence of slopes converges, $\lim \mu_{\alpha}\left(\mathscr{F}_{i}\right)=\mu_{\alpha}^{\max }(\mathscr{E})$.

(2.23.2) The sheaves $\mathscr{F}_{i}$ are saturated in $\mathscr{E}$. All $\mathscr{F}_{i}$ have the same rank $r$.

In addition, we can assume that the rank $r$ is maximal among all sequences of sheaves satisfying (2.23.1)-(2.23.2). We will construct a contradiction by proving the following.

Given any number $\varepsilon>0$, there exists a subsheaf $\mathscr{G}_{\varepsilon} \subseteq \mathscr{E}$ such that $\mu_{\alpha}\left(\mathscr{G}_{\varepsilon}\right) \geq \mu_{\alpha}^{\max }(\mathscr{E})-\varepsilon$ and such that $\operatorname{rank} \mathscr{G}_{\varepsilon}>r$.

To this end, let $\varepsilon$ be any given number. Then, there exists an index $i$ such that $\mu_{\alpha}\left(\mathscr{F}_{i}\right)>\mu_{\alpha}^{\max }(\mathscr{E})-\frac{\varepsilon}{2}$. There exists a larger index $j>i$ such that $\mu_{\alpha}\left(\mathscr{F}_{i}\right)<$ $\mu_{\alpha}\left(\mathscr{F}_{j}\right)$. The subsheaves $\mathscr{F}_{i}$ and $\mathscr{F}_{j}$ are certainly not equal. Since both $\mathscr{F}_{i}$ and $\mathscr{F}_{j}$ are saturated in $\mathscr{E}$, their sum $\mathscr{G}_{\varepsilon}:=\mathscr{F}_{i}+\mathscr{F}_{j}$ therefore has rank $\mathscr{G}_{\varepsilon}>r$. A rather elementary computation of Chern classes, spelled out in [GKP14, Lemma A.12], now shows that $\mu_{\alpha}\left(\mathscr{G}_{\varepsilon}\right)>\mu_{\alpha}^{\max }(\mathscr{E})-\varepsilon$.

Remark 2.24. Any sheaf $\mathscr{F} \subseteq \mathscr{E}$ with $\mu_{\alpha}^{\max }(\mathscr{E})=\mu_{\alpha}(\mathscr{F})$ is clearly semistable.

2.6. Existence of the Harder-Narasimhan-filtration. One consequence of the boundedness result obtained in Proposition 2.23 is the existence of a maximally destabilising subsheaf, which in turn implies the existence of the HarderNarasimhan and of Jordan-Hölder-filtrations, even in cases where slope is defined by a movable curve class.

Corollary 2.25 (Existence of a unique maximally destabilising sheaf). In the setting of Definition 2.21, there exists a sheaf $\mathscr{F} \subseteq \mathscr{E}$ such that the following holds.

(2.25.1) The slope is maximal, $\mu_{\alpha}(\mathscr{F})=\mu_{\alpha}^{\max }(\mathscr{E})$.

(2.25.2) If $\mathscr{F}^{\prime} \subseteq \mathscr{E}$ is any other subsheaf with $\mu_{\alpha}(\mathscr{F})=\mu_{\alpha}^{\max }(\mathscr{E})$, then $\mathscr{F}^{\prime} \subseteq \mathscr{F}$.

The sheaf $\mathscr{F}$ is called "maximally destabilising subsheaf". It is clearly unique, semistable, and saturated in $\mathscr{E}$.

Proof. By Proposition 2.23, there exists a saturated sheaf $\mathscr{F}_{1} \subseteq \mathscr{E}$ of maximal slope. If $\mathscr{F}^{\prime}$ is any other subsheaf of maximal slope, then either $\mathscr{F}^{\prime}$ is contained in $\mathscr{F}_{1}$, or it follows from Proposition 2.17 that the $\alpha$-slope of $\mathscr{F}_{1}+\mathscr{F}^{\prime}$ equals $\mu_{\alpha}^{\max }(\mathscr{E})$. In the second case, set $\mathscr{F}_{2}:=\left(\mathscr{F}_{1}+\mathscr{F}^{\prime}\right)_{\text {sat }}$ and observe that rank $\mathscr{F}_{2}>\operatorname{rank} \mathscr{F}_{1}$. Iterate this process, in order to construct a strictly increasing sequence of sheaves of maximal slope, $\mathscr{F}_{1} \subsetneq \mathscr{F}_{2} \subsetneq \ldots \subseteq \mathscr{E}$. The process terminates because the rank increases in each step.

Corollary 2.26 (Existence of a stable destabilising sheaf). In the setting of Definition 2.21, there exists an $\alpha$-stable sheaf $\mathscr{F} \subseteq \mathscr{E}$ of slope $\mu_{\alpha}(\mathscr{F})=\mu_{\alpha}^{\max }(\mathscr{E})$.

Proof. By Proposition 2.23, there exists a saturated, $\alpha$-semistable sheaf $\mathscr{F}_{1} \subseteq \mathscr{E}$ of maximal slope. If $\mathscr{F}_{1}$ is not stable, there exists a sheaf $\mathscr{F}_{2} \subsetneq \mathscr{F}_{1}$ that is also of maximal slope, but of smaller rank: rank $\mathscr{F}_{2}<\operatorname{rank} \mathscr{F}_{1}$. Iterate this process, 
in order to construct a strictly decreasing sequence of sheaves of maximal slope, $\mathscr{F}_{1} \supsetneq \mathscr{F}_{2} \supsetneq \cdots$. The process terminates because the rank decreases in each step.

Corollary 2.27 (Existence of the Harder-Narasimhan-filtration). In the setting of Definition 2.21, there exists a unique "Harder-Narasimhan-filtration", that is, a filtration $0=\mathscr{E}_{0} \subsetneq \mathscr{E}_{1} \subsetneq \cdots \subsetneq \mathscr{E}_{r}=\mathscr{E}$ where each quotient $\mathscr{Q}_{i}:=\mathscr{E}_{i} / \mathscr{E}_{i-1}$ is torsion-free, $\alpha$-semistable, and where the sequence of slopes $\mu_{\alpha}\left(\mathscr{Q}_{i}\right)$ is strictly decreasing.

Corollary 2.28 (Existence of Jördan-Hölder-filtrations). In the setting of Definition 2.21, if $\mathscr{E}$ is $\alpha$-semistable, then there exists a "Jordan-Hölder-filtration", that is, a filtration $0=\mathscr{E}_{0} \subsetneq \mathscr{E}_{1} \subsetneq \cdots \subsetneq \mathscr{E}_{r}=\mathscr{E}$ where each quotient $\mathscr{Q}_{i}:=\mathscr{E}_{i} / \mathscr{E}_{i-1}$ is torsionfree, $\alpha$-stable, and with slopes $\mu_{\alpha}\left(\mathscr{Q}_{i}\right)=\mu_{\alpha}(\mathscr{E})$.

Remark 2.29 (Refined Harder-Narasimhan-filtration). In the setting of Definition 2.21, combining Harder-Narasimhan and Jordan-Hölder filtrations, one obtains a "refined Harder-Narasimhan-filtration" $0=\mathscr{E}_{0} \subsetneq \mathscr{E}_{1} \subsetneq \cdots \subsetneq \mathscr{E}_{r}=\mathscr{E}$ where each quotient $\mathscr{Q}_{i}:=\mathscr{E}_{i} / \mathscr{E}_{i-1}$ is torsion-free, $\alpha$-stable, and where the sequence of slopes $\mu_{\alpha}\left(\mathscr{Q}_{i}\right)$ is decreasing (though not necessarily strictly decreasing).

2.7. Boundedness II: Grothendieck's lemma. Let $X$ be a projective manifold and $\mathscr{E}$ be any torsion-free sheaf on $X$. If $H$ any ample Cartier divisor with associated movable curve class $\beta:=H^{\operatorname{dim} X-1}$ and $c$ is any real number, then the classical Grothendieck lemma, [HL10, Lem. 1.7.9], asserts that the set of subsheaves with bounded slope,

$$
S:=\left\{\mathscr{F} \mid \mathscr{F} \subseteq \mathscr{E} \text { saturated, positive-rank with } \mu_{\beta}(\mathscr{F}) \geq c\right\}
$$

forms a bounded family. In particular, the associated set of numerical classes is finite. We show that this conclusion still holds in case where $\beta$ is an arbitrary big class.

Theorem 2.30 (Grothendieck's lemma for numerical classes). Let $X$ be a normal, projective, $Q$-factorial variety and $\beta \in \operatorname{Mov}(X)$ be a big class. Further, let $\mathscr{E}$ be a torsionfree, coherent sheaf on $X$ and $c \in \mathbb{R}$ be a real number. Then, the following set of numerical classes,

$$
S_{\text {num }}:=\left\{[\mathscr{F}] \mid \mathscr{F} \subseteq \mathscr{E} \text { any positive-rank subsheaf with } \mu_{\beta}(\mathscr{F}) \geq c\right\} \subseteq N^{1}(X)_{\mathbb{Q}},
$$

is finite.

Proof. As before, choose a sufficiently ample bundle $\mathscr{H}$ and an embedding $\mathscr{E} \hookrightarrow$ $\mathscr{H}^{\oplus r}$, where $r:=\operatorname{rank} \mathscr{E}$. It will then suffice to show the claim in case where $\mathscr{E}=\mathscr{H}^{\oplus r}$. Since slopes behave linearly under twist, we may even assume without loss of generality that $\mathscr{E}=\mathscr{O}_{X}^{\oplus r}$.

Now, given any subsheaf $\mathscr{F} \subseteq \mathscr{E}$ of positive rank, its determinant $\operatorname{det} \mathscr{F}$ embeds into $\mathscr{O}_{X}$ and is therefore a Weil divisorial sheaf of the form det $\mathscr{F} \cong \mathscr{O}_{X}(-D)$, where $D$ is an effective, integral Weil divisor. Since $\beta$ is assumed to be a big class, it will therefore intersect $[\mathscr{F}]$ negatively, unless $[\mathscr{F}]=0$. The assertion is thus a consequence of Theorem 2.7.

\section{OPENNESS OF SEMISTABILITY}

We now show that stability is an open property, at least within the interior of the movable cone. More precisely, we discuss openness properties for the set of stabilising classes, defined as follows. 


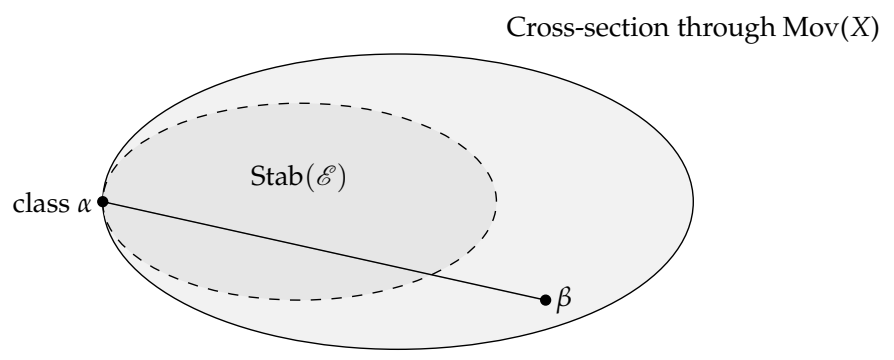

\begin{abstract}
The set of movable classes that stabilise a given bundle $\mathscr{E}$ is open in the interior of the movable cone, but does contain an isolated class on the boundary. Note that Theorem 3.4

holds in this context because the boundary of $\operatorname{Stab}(\mathscr{E})$ intersects the boundary of the movable cone tangentially.
\end{abstract}

FIGURE 3.1. Hypothetical cross-section through $\operatorname{Mov}(X)$.

Definition 3.1 (Stabilising classes). Let X be a normal, Q-factorial, projective variety and let $\mathscr{E}$ be any non-trivial, torsion-free sheaf on $X$. We consider the set of movable classes that (semi-)stabilise $\mathscr{E}$,

$$
\begin{aligned}
\operatorname{Stab}(\mathscr{E}) & :=\{\alpha \in \operatorname{Mov}(X) \mid \mathscr{E} \text { is } \alpha \text {-stable }\} \\
\operatorname{SStab}(\mathscr{E}) & :=\{\alpha \in \operatorname{Mov}(X) \mid \mathscr{E} \text { is } \alpha \text {-semistable }\} .
\end{aligned}
$$

Remark 3.2 (Convexity of stabilising classes). The sets $\operatorname{Stab}(\mathscr{E})$ and $\operatorname{SStab}(\mathscr{E})$ of Definition 3.1 are clearly convex. The set $\operatorname{SStab}(\mathscr{E})$ is closed and contains $\operatorname{Stab}(\mathscr{E})$.

Theorem 3.3 (Openness of stability, I). Let X be a normal, Q-factorial, projective variety and let $\mathscr{E}$ be any non-trivial, torsion-free sheaf on $X$. If $\alpha \in \operatorname{Stab}(\mathscr{E})$ is big, then $\operatorname{Stab}(\mathscr{E})$ contains an open neighbourhood $U=U(\alpha) \subseteq \operatorname{Mov}(X)$.

The following result asserts that even in cases where we cannot show openness, any class $\alpha \in \operatorname{Stab}(\mathscr{E})$ can be approximated by big classes in $\operatorname{Stab}(\mathscr{E})$. The authors would like to thank Matei Toma who explained Theorem 3.4 to us in case where $\beta$ is a general complete intersection curve. Together with further related results, his argument will also appear in [GRT14].

Theorem 3.4 (Openness of stability, II). Let X be a normal, Q-factorial, projective variety and let $\mathscr{E}$ be any non-trivial, torsion-free sheaf on X. Consider classes $\alpha \in \operatorname{Stab}(\mathscr{E})$ and $\beta \in \operatorname{Mov}(X)^{\circ}$. Then, there exists a number $e \in \mathbb{Q}^{+}$such that $(\alpha+\varepsilon \cdot \beta) \in \operatorname{Stab}(\mathscr{E})$, for any real $\varepsilon \in[0, e]$. In particular, if $L \subset N_{1}(X)_{\mathbb{R}}$ is any line through $\alpha$ that intersects the interior $\operatorname{Mov}(X)^{\circ}$, then $L \cap \operatorname{Stab}(\mathscr{E})$ is an interval of positive length.

Remark 3.5. Let $X$ be a normal, Q-factorial, projective variety and let $\mathscr{E}$ be any non-trivial, torsion-free sheaf on $X$. Given any class $\alpha \in \operatorname{Stab}(\mathscr{E})$, Theorems 3.3 and 3.4 together assert that $\operatorname{Stab}(\mathscr{E}) \cap \operatorname{Mov}(X)^{\circ}$ is open and has $\alpha$ as a boundary point. In particular, there exists a sequence of big, rational classes $\beta_{i} \in \operatorname{Stab}(\mathscr{E})$ with $\lim \beta_{i}=\alpha$.

We do not know if these results are optimal. For instance, we cannot rule out that the set of movable classes that stabilise a given bundle $\mathscr{E}$ is of the form illustrated in Figure 3.1. For all applications, Theorems 3.3 and 3.4 seem to suffice.

Question 3.6. Are there examples where $\operatorname{Stab}(\mathscr{E})$ is not open in $\operatorname{Mov}(X)$ ? If so, are there natural conditions to guarantee openness?

We begin with a preparatory subsection. Theorems 3.3 and 3.4 are then shown in Sections 3.2-3.3 below. 
3.1. Suprema of slopes of strict subsheaves. Given a sheaf $\mathscr{E}$ and a movable class $\alpha$, we have discussed the number $\mu_{\alpha}^{\max }(\mathscr{E})$ in Section 2.5. Here, we discuss a similar (but more delicate) quantity, namely the supremum of slopes of subsheaves $\mathscr{F} \subsetneq \mathscr{E}$ whose rank is strictly smaller than that of $\mathscr{E}$. If $\alpha$ is either big or rational, we will again see that the supremum is in fact a maximum.

Definition 3.7 (Suprema of slopes of strict subsheaves). Let $X$ be a normal, Qfactorial, projective variety and $\alpha \in \operatorname{Mov}(X)$ a movable class. If $\mathscr{E}$ is any torsion-free, coherent sheaf of $\mathscr{O}_{X}$-modules with rank $\mathscr{E} \geq 2$, write

$$
\mu_{\alpha}^{\max , s c}(\mathscr{E}):=\sup \left\{\mu_{\alpha}(\mathscr{F}) \mid 0 \neq \mathscr{F} \subsetneq \mathscr{E} \text { coherent with rank } \mathscr{F}<\operatorname{rank} \mathscr{E}\right\} .
$$

Remark 3.8. Obviously, $\mu_{\alpha}^{\max , s c}(\mathscr{E}) \leq \mu_{\alpha}^{\max }(\mathscr{E})$.

Proposition 3.9 (Existence of subsheaves with maximal slope, II). In the setting of Definition 3.7, suppose that $\alpha$ is big or that $\alpha$ is rational. Then, there exists a coherent subsheaf $\mathscr{F} \subseteq \mathscr{E}$ such that rank $\mathscr{F}<\operatorname{rank} \mathscr{E}$ and such that $\mu_{\alpha}^{\max , s c}(\mathscr{E})=\mu_{\alpha}(\mathscr{F})$.

Proof of Proposition 3.9 in case where $\alpha$ is big. Observing that the statement of Proposition 3.9 remains invariant when twisting $\mathscr{E}$, we may replace $\mathscr{E}$ by a tensor product and assume that there exists a number $N \in \mathbb{N}^{+}$, an ample line bundle $\mathscr{H}$ and a surjection $\mathscr{H}^{\oplus N} \rightarrow \mathscr{E}$. In particular, given any torsion-free quotient $\mathscr{E} \rightarrow \mathscr{Q}$ of positive rank, there exists a non-trivial morphism of Weil divisorial sheaves

$$
\mathscr{H}^{\otimes \operatorname{rank} \mathscr{Q}} \rightarrow \operatorname{det} \mathscr{Q} .
$$

It follows that the numerical class [Q2] is pseudo-effective, and in fact big.

Introduce any norm $\|\cdot\|$ on the finite-dimensional space $N^{1}(X)_{\mathbb{R}}$ of numerical Cartier divisor classes. Since $\alpha$ is big, there exists a constant $C>0$ such that

$$
D \cdot \alpha \geq C \cdot\|D\| \quad \text { for any pseudo-effective } D \in N^{1}(X)_{\mathbb{R}} .
$$

In particular, Inequality (3.9.1) holds for divisors $D$ that represent numerical classes of non-trivial, torsion-free quotients of $\mathscr{E}$.

Returning to the assertion of Proposition 3.9, we argue by contradiction and assume that the number $\mu_{\alpha}^{\max , s c}(\mathscr{E})$ is not attained. Then, there exists a sequence of subsheaves $\mathscr{F}_{j} \subsetneq \mathscr{E}$ such that the sequence of slopes $\mu_{\alpha}\left(\mathscr{F}_{j}\right)$ is strictly increasing and converges to $\mu_{\alpha}^{\max , s c}(\mathscr{E})$. Corollary 2.14 allows to assume that the quotients $\mathscr{Q}_{j}:=\mathscr{E} / \mathscr{F}_{j}$ are torsion-free.

The assumption that $\mu_{\alpha}\left(\mathscr{F}_{j}\right)$ is strictly increasing implies that the sets of numerical classes $\left\{\left[\mathscr{F}_{j}\right] \mid j \in \mathbb{N}\right\}$ and $\left\{\left[\mathscr{Q}_{j}\right] \mid j \in \mathbb{N}\right\}$ are both infinite. The theorem on finite generation of numerical divisor classes, Theorem 2.7, thus implies that both sets are unbounded with respect to the norm $\|\cdot\|$. Inequality (3.9.1) thus implies that the sequence $\left(\mu_{\alpha}\left(\mathscr{Q}_{j}\right)\right)_{j \in \mathbb{N}}$ is unbounded, and so is $\left(\mu_{\alpha}\left(\mathscr{F}_{j}\right)\right)_{j \in \mathbb{N}}$. This contradicts convergence.

Proof of Proposition 3.9 in case where $\alpha$ is rational. If $\alpha$ is rational, the Theorem on finite generation of numerical divisor classes, Theorem 2.7, allows to find an integer $m \in \mathbb{N}^{+}$such that $(m \cdot \alpha) \cdot[\mathscr{F}]$ is integral, for any subsheaf $\mathscr{F} \subseteq \mathscr{E}$ of positive rank (and in fact for any coherent sheaf on $X)$. It follows that the slope $\mu_{\alpha}(\mathscr{F})$ takes values in the discrete set $\frac{1}{(\operatorname{rank} \mathscr{E}) ! \cdot m} \cdot \mathbb{Z}$ and the claim is obvious.

Remark 3.10. Let $\alpha$ be a movable class that is either big or rational. As a consequence of Proposition 3.9, we see that $\mathscr{E}$ is $\alpha$-stable if and only if $\mu_{\alpha}^{\max , s c}(\mathscr{E})<$ $\mu_{\alpha}^{\max }(\mathscr{E})$. 
Remark 3.11. There are relevant situations where the conclusion of Proposition 3.9 holds even for irrational classes that lie on the boundary of $\operatorname{Mov}(X)$. The most significant is that where $\pi: \widetilde{X} \rightarrow X$ is a resolution of singularities and $\alpha \in \operatorname{Mov}(X)$ a big, movable class. Its pull-back $\pi^{*} \alpha$ is a movable class contained in the boundary $\partial \operatorname{Mov}(\widetilde{X})$. However, Proposition 2.8 guarantees that everything said so far also holds for $\phi^{*} \alpha$.

Remark 3.12. We do not know whether Proposition 3.9 remains true for irrational classes $\alpha$ that lie on the boundary of the movable cone.

3.2. Proof of Theorem 3.3. Observe that if $\operatorname{rank} \mathscr{E}=1$, then there is nothing to show. We will therefore assume throughout that proof that rank $\mathscr{E} \geq 2$. Choose any relatively compact, open neighbourhood of $\alpha$ in $\operatorname{Mov}(X)$, say $V=V(\alpha)$. Then, there exists a (small) number $e \in \mathbb{Q}^{+}$such that the following holds for all numbers $0 \leq \varepsilon \leq e$ and for all classes $\beta \in V$,

$$
\varepsilon \cdot(\underbrace{\mu_{\beta}^{\max }(\mathscr{E})-\mu_{\beta}(\mathscr{E})}_{\text {bounded on } V \text { by Prop. } 2.22})<(1-\varepsilon) \cdot(\underbrace{\mu_{\alpha}(\mathscr{E})-\mu_{\alpha}^{\max , s c}(\mathscr{E})}_{>0 \text { by Prop. } 3.9}) .
$$

The following will then hold for any class $\beta \in V$, any coherent subsheaf $\mathscr{F} \subsetneq \mathscr{E}$ with $\operatorname{rank} \mathscr{F}<\operatorname{rank} \mathscr{E}$, and any number $0<\varepsilon \leq e$,

$$
\begin{aligned}
\mu_{(1-\varepsilon) \cdot \alpha+\varepsilon \cdot \beta}(\mathscr{F}) & =(1-\varepsilon) \cdot \mu_{\alpha}(\mathscr{F})+\varepsilon \cdot \mu_{\beta}(\mathscr{F}) \\
& \leq(1-\varepsilon) \cdot \mu_{\alpha}^{\max , s c}(\mathscr{E})+\varepsilon \cdot \mu_{\beta}^{\max }(\mathscr{E}) \\
& <(1-\varepsilon) \cdot \mu_{\alpha}(\mathscr{E})+\varepsilon \cdot \mu_{\beta}(\mathscr{E}) \\
& =\mu_{(1-\varepsilon) \cdot \alpha+\varepsilon \cdot \beta}(\mathscr{E}) .
\end{aligned}
$$

Finish the proof of Theorem 3.3 by setting $U:=e \cdot(V-\alpha)+\alpha$.

3.3. Proof of Theorem 3.4. Consider the set $S$ of classes $[\mathscr{F}]$ of sheaves $\mathscr{F} \subseteq$ $\mathscr{E}$ that destabilise $\mathscr{E}$ with respect to $\beta$, i.e., $\mu_{\beta}(\mathscr{F})>\mu_{\beta}(\mathscr{E})$. By Grothendieck's lemma for numerical classes, Theorem 2.30, the set $S$ is finite, say $S=$ $\left\{\left[\mathscr{F}_{1}\right], \ldots,\left[\mathscr{F}_{n}\right]\right\}$ for suitable $\mathscr{F}_{i} \subseteq \mathscr{E}$. Now, if $\mathscr{F} \subset \mathscr{E}$ is any subsheaf with rank $\mathscr{F}<$ rank $\mathscr{E}$, there are two cases:

- $[\mathscr{F}] \notin S$ and it follows from $\alpha$-stability of $\mathscr{E}$ that

$$
\mu_{(1-\varepsilon) \cdot \alpha+\varepsilon \cdot \beta}(\mathscr{F})<\mu_{(1-\varepsilon) \cdot \alpha+\varepsilon \cdot \beta}(\mathscr{E}) \quad \text { for all } \varepsilon \in[0,1)
$$

- $[\mathscr{F}] \in S$ and there exists an index $j$ such that $[\mathscr{F}]=\left[\mathscr{F}_{j}\right]$, hence

$$
\mu_{(1-\varepsilon) \cdot \alpha+\varepsilon \cdot \beta}(\mathscr{F})=\mu_{(1-\varepsilon) \cdot \alpha+\varepsilon \cdot \beta}\left(\mathscr{F}_{j}\right) \text { for all } \varepsilon \in[0,1] .
$$

In either case, we obtain the following inequality for all $\varepsilon \in[0,1]$,

$$
\mu_{(1-\varepsilon) \cdot \alpha+\varepsilon \cdot \beta}(\mathscr{F}) \leq \max \{\underbrace{\max _{1 \leq j \leq n} \mu_{(1-\varepsilon) \cdot \alpha+\varepsilon \cdot \beta}\left(\mathscr{F}_{j}\right)}_{=: \Phi(\varepsilon)}, \mu_{(1-\varepsilon) \cdot \alpha+\varepsilon \cdot \beta}(\mathscr{E})\} .
$$

The inequality is strict for all $\varepsilon$ for which $\Phi(\varepsilon)<\mu_{(1-\varepsilon) \cdot \alpha+\varepsilon \cdot \beta}(\mathscr{E})$. The setup is depicted in Figure 3.2 on the next page. Conclude by observing that $\Phi$ is continuous as a function of $\varepsilon$, and that $\Phi(0)<\mu_{\alpha}(\mathscr{E})$.

\section{TENSOR PRODUCTS OF SEMISTABLE SHEAVES}

In this section we prove that the reflexive tensor product of sheaves which are $\alpha$ semistable for some movable class $\alpha$ is again $\alpha$-semistable. The following notation will be used. 


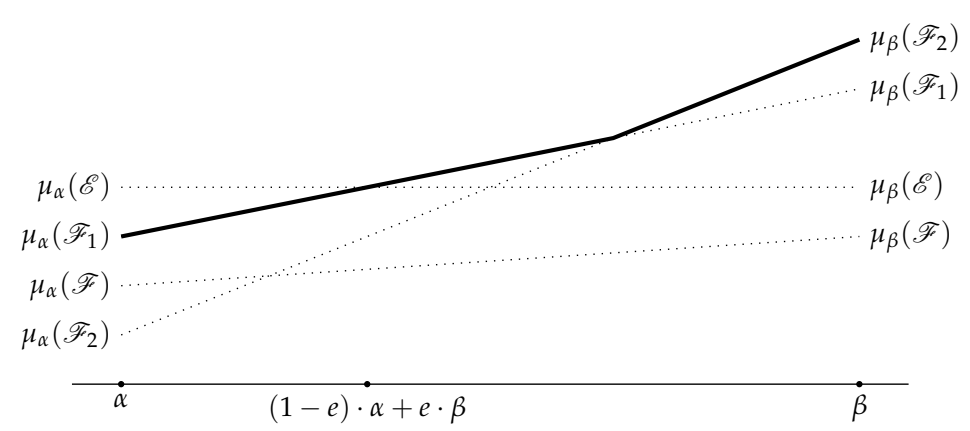

Slopes of the sheaves $\mathscr{F}_{j}$ and of a sheaf $\mathscr{F}$ such that $[\mathscr{F}] \notin S$ with respect to movable classes $(1-\varepsilon) \cdot \alpha+\varepsilon \cdot \beta$. The function $\Phi$ is outlined in bold.

FIGURE 3.2. Functions discussed in the proof of Theorem 3.4

Notation 4.1 (Reflexive tensor product). Given any two coherent sheaves $\mathscr{A}, \mathscr{B}$ of $\mathscr{O}_{\mathrm{X}}$-modules on a normal variety $\mathrm{X}$, write $\mathscr{A} \otimes \mathscr{B}:=(\mathscr{A} \otimes \mathscr{B})^{* *}$. We refer to $\mathscr{A} \otimes \mathscr{B}$ as the reflexive tensor product of $\mathscr{A}$ and $\mathscr{B}$.

Theorem 4.2 (Semistability of tensor products). Let X be a normal Q-factorial projective variety, and let $\alpha \in \operatorname{Mov}(X)$ be any movable class. If $\mathscr{F}$ and $\mathscr{G}$ are torsion-free, positive-rank, coherent sheaves on $X$, then the following holds.

(4.2.1) $\mu_{\alpha}^{\max }(\mathscr{F} \otimes \mathscr{G})=\mu_{\alpha}^{\max }(\mathscr{F})+\mu_{\alpha}^{\max }(\mathscr{G})$.

(4.2.2) If $\mathscr{F}$ and $\mathscr{G}$ are $\alpha$-semistable, then $\mathscr{F} \otimes \mathscr{G}$ is likewise $\alpha$-semistable.

Corollary 4.3 (Semistability of reflexive symmetric products). In the setting of Theorem 4.2 , if $\mathscr{F}$ is $\alpha$-semistable and $m \in \mathbb{N}^{+}$any number, then any reflexive symmetric product $\left(\mathrm{Sym}^{m} \mathscr{F}\right)^{* *}$ is again $\alpha$-semistable.

Proof. Observe that $\left(\mathrm{Sym}^{m} \mathscr{F}\right)^{* *}$ is a direct summand of $\mathscr{F} \otimes m$.

4.1. Preparation for the proof: reduction to semistability of tensor products of stable sheaves. We show in this section that to prove Theorem 4.2, it suffices to show that the product of two stable sheaves is semistable. The following is the main result of the present Section 4.1.

Proposition 4.4. Let X be a normal, $Q$-factorial, projective variety and let $\alpha \in \operatorname{Mov}(X)$ be any movable class. Suppose that the reflexive tensor product of any two torsion-free, $\alpha$-stable sheaves is $\alpha$-semistable. Then, the following holds for any two torsion-free sheaves $\mathscr{F}$ and $\mathscr{G}$ on $X$.

(4.4.1) $\mu_{\alpha}^{\max }(\mathscr{F} \otimes \mathscr{G})=\mu_{\alpha}^{\max }(\mathscr{F})+\mu_{\alpha}^{\max }(\mathscr{G})$.

(4.4.2) If $\mathscr{F}$ and $\mathscr{G}$ are $\alpha$-semistable, then $\mathscr{F} \otimes \mathscr{G}$ is again $\alpha$-semistable.

We prove Proposition 4.4 in the remainder of Section 4.1. The proof is subdivided into five steps.

Step 1: Setup. Since numerical classes and slopes are unaffected when modifying $\mathscr{F}$ and $\mathscr{G}$ along a subset of codimension at least two, we are free to replace these sheaves by their double duals, and assume henceforth that the following holds.

Assumption w.l.o.g. 4.5. The sheaves $\mathscr{F}$ and $\mathscr{G}$ are reflexive.

Combining the Harder-Narasimhan filtration and a Jordan-Hölder-filtration as in Remark 2.29, choose a filtration of $\mathscr{F}$, say $0=\mathscr{F}_{0} \subsetneq \mathscr{F}_{1} \subsetneq \cdots \subsetneq \mathscr{F}_{k}=\mathscr{F}$ such that the following holds. 
(4.6.1) The quotients $\mathscr{Q}_{i+1}:=\mathscr{F}_{i+1} / \mathscr{F}_{i}$ are torsion-free and $\alpha$-stable for all $i$.

(4.6.2) The sequence of ranks, $\left(\text { rank } \mathscr{F}_{i}\right)_{0 \leq i \leq k}$, is strictly increasing.

(4.6.3) The sequence of slopes, $\left(\mu_{\alpha}\left(\mathscr{Q}_{i}\right)\right)_{1 \leq i \leq k^{\prime}}$ is decreasing.

Taking reflexive tensor products with $\mathscr{G}$, we obtain a filtration of $\mathscr{F} \otimes \mathscr{G}$,

$$
0=\mathscr{F}_{0} \otimes \mathscr{G} \subsetneq \mathscr{F}_{1} \otimes \mathscr{G} \subsetneq \cdots \subsetneq \mathscr{F}_{k} \otimes \mathscr{G}=\mathscr{F} \otimes \mathscr{G},
$$

where each term $\mathscr{F}_{i} \otimes \mathscr{G}$ is saturated in $\mathscr{F}_{i+1} \otimes \mathscr{G}$. Consequently there exists a big, open subset $X^{\circ} \subseteq X$ where all sheaves $\mathscr{F}, \mathscr{G}, \mathscr{F}_{i}$ and $\mathscr{F}_{i} \otimes \mathscr{G}$, as well as all quotients $\mathscr{Q}_{i}$ and $\left(\mathscr{F}_{i+1} \otimes \mathscr{G}\right) /\left(\mathscr{F}_{i} \otimes \mathscr{G}\right)$ are locally free. Since two reflexive sheaves are isomorphic if and only if they agree over $X^{\circ}$, the quotients given by the filtration (4.6.4) can be identified as follows,

$$
\left(\mathscr{F}_{i+1} \otimes \mathscr{G} / \mathscr{F}_{i} \otimes \mathscr{G}\right)^{* *}=\mathscr{Q}_{i+1} \otimes \mathscr{G} .
$$

The slopes of these sheaves are computed as follows.

$$
\begin{array}{rlrl}
\mu_{\alpha}\left(\mathscr{Q}_{i+1} \nabla \mathscr{G}\right) & =\mu_{\alpha}\left(\mathscr{Q}_{i+1}\right)+\mu_{\alpha}(\mathscr{G}) & & \text { Slope of product } \\
& \leq \mu_{\alpha}\left(\mathscr{Q}_{1}\right)+\mu_{\alpha}(\mathscr{G}) & & \text { by }(4.6 .3) \\
& \leq \mu_{\alpha}^{\max }(\mathscr{F})+\mu_{\alpha}^{\max }(\mathscr{G}) &
\end{array}
$$

Step 2: Proof of Claim (4.4.1) in case where $\mathscr{F}$ or $\mathscr{G}$ are $\alpha$-stable. The roles of $\mathscr{F}$ and $\mathscr{G}$ being symmetric, consider the case where $\mathscr{G}$ is $\alpha$-stable. By assumption, the quotient sheaves $\mathscr{Q}_{i} \otimes \mathscr{G}$ will thus be $\alpha$-semistable. Inequality (4.6.6) therefore implies that any morphism $\mathscr{A} \rightarrow \mathscr{Q}_{i} \otimes \mathscr{G}$ will be zero, if $\mathscr{A}$ is any semistable subsheaf of slope $\mu_{\alpha}(\mathscr{A})>\mu_{\alpha}^{\max }(\mathscr{F})+\mu_{\alpha}^{\max }(\mathscr{G})$. It follows that any morphism $\mathscr{A} \rightarrow \mathscr{F} \otimes \mathscr{G}$ will be zero, and that the $\alpha$-slope of any semistable subsheaf $\mathscr{B} \subseteq$ $\mathscr{F} \nabla \mathscr{G}$ is bounded. In other words, we have

$$
\mu_{\alpha}(\mathscr{B}) \leq \mu_{\alpha}^{\max }(\mathscr{F})+\mu_{\alpha}^{\max }(\mathscr{G}) .
$$

On the other hand, $\mathscr{F} \otimes \mathscr{G}$ does contain the semistable subsheaf $\mathscr{F}_{1} \otimes \mathscr{G}=\mathscr{Q}_{1} \otimes$ $\mathscr{G}$, whose slope equals $\mu_{\alpha}^{\max }(\mathscr{F})+\mu_{\alpha}(\mathscr{G})=\mu_{\alpha}^{\max }(\mathscr{F})+\mu_{\alpha}^{\max }(\mathscr{G})$. This proves Claim (4.4.1) in case where one of the factors is $\alpha$-stable.

Step 3: Proof of Claim (4.4.1) in general. Recalling from (4.6.1) that quotient sheaves $\mathscr{Q}_{i+1}$ are $\alpha$-stable, we have seen in Step 2 that

$$
\mu_{\alpha}^{\max }\left(\mathscr{Q}_{i+1} \nabla \mathscr{G}\right)=\mu_{\alpha}^{\max }\left(\mathscr{Q}_{i+1}\right)+\mu_{\alpha}^{\max }(\mathscr{G}) \leq \mu_{\alpha}^{\max }(\mathscr{F})+\mu_{\alpha}^{\max }(\mathscr{G}) .
$$

As in Step 2, this implies that the $\alpha$-slope of any subsheaf $\mathscr{B} \subseteq \mathscr{F} \otimes \mathscr{G}$ is bounded by $\mu_{\alpha}^{\max }(\mathscr{F})+\mu_{\alpha}^{\max }(\mathscr{G})$. On the other hand, $\mathscr{F} \otimes \mathscr{G}$ does contain the reflexive product of the maximally destabilising subsheaves. The slope of this product equals $\mu_{\alpha}^{\max }(\mathscr{F})+\mu_{\alpha}^{\max }(\mathscr{G})$. This proves Claim (4.4.1) in general.

Step 4: Proof of Claim (4.4.2). Now assume that $\mathscr{F}$ and $\mathscr{G}$ are both $\alpha$-semistable. The slope of the product is computed as follows,

$$
\begin{aligned}
\mu_{\alpha}^{\max }(\mathscr{F} \otimes \mathscr{G}) & =\mu_{\alpha}^{\max }(\mathscr{F})+\mu_{\alpha}^{\max }(\mathscr{G}) & & \text { Claim }(4.4 .1) \\
& =\mu_{\alpha}(\mathscr{F})+\mu_{\alpha}(\mathscr{G}) & & \text { Semistability of } \mathscr{F} \text { and } \mathscr{G} \\
& =\mu_{\alpha}(\mathscr{F} \otimes \mathscr{G}), & &
\end{aligned}
$$

proving its semistability. This proves Claim (4.4.2) and finishes the proof of Proposition 4.4. 
4.2. Reduction to a resolution. We end the preparations for the proof of Theorem 4.2 with the following lemma. Both its claims follow immediately from Proposition 2.8 .

Lemma 4.7. In the setting of Theorem 4.2 , let $\pi: \widetilde{X} \rightarrow X$ be any resolution of singularities. Then the following holds.

(4.7.1) The sheaf $\mathscr{F}$ is $\alpha$-stable if and only if $\pi^{[*]} \mathscr{F}$ is $\left(\pi^{*} \alpha\right)$-stable.

(4.7.2) The sheaf $\mathscr{F} \otimes \mathscr{G}$ is $\alpha$-semistable if and only if $\pi^{[*]} \mathscr{F} \otimes \pi^{[*]} \mathscr{G}$ is $\left(\pi^{*} \alpha\right)$ semistable.

4.3. Proof of Theorem 4.2. We have seen in Proposition 4.4 that to prove Theorem 4.2, it suffices to show that the reflexive tensor product of any two $\alpha$-stable sheaves is $\alpha$-semistable. So, let $\mathscr{F}$ and $\mathscr{G}$ be any two $\alpha$-stable, torsion-free, coherent sheaves on $X$.

Combining a resolution of singularities with a classical result of Rossi, [Ros68], we find a smooth, projective variety $\widetilde{X}$ and a birational morphism $\pi: \widetilde{X} \rightarrow X$ such that $\pi^{[*]} \mathscr{F}$ and $\pi^{[*]} \mathscr{G}$ are both locally free. By Lemma 4.7, it suffices to establish $\left(\pi^{*} \alpha\right)$-semistability of $\pi^{[*] \mathscr{F}} \otimes \pi^{[*]} \mathscr{G}$. To simplify notation we replace $X$ by $\widetilde{X}$ and assume without loss of generality that the following holds.

Assumption w.l.o.g. 4.8. The variety $X$ is smooth. The sheaves $\mathscr{F}$ and $\mathscr{G}$ are locally free.

Under these assumptions, Theorem 4.2 has been shown by Toma in [CP11, Prop. 6.1] if the class $\alpha$ is rational and big. To show Theorem 4.2 in the general case, let $\mathscr{A} \subset \mathscr{F} \otimes \mathscr{G}$ be any proper subsheaf. Recall from Remark 3.5 that we can find a sequence of big, rational classes $\beta_{i} \in \operatorname{Stab}(\mathscr{F}) \cap \operatorname{Stab}(\mathscr{G})$ with $\lim \beta_{i}=\alpha$. Toma's result [CP11, Prop. 6.1] applies to show that $\mathscr{F} \otimes \mathscr{G}$ is stable with respect to the $\beta_{i}$, and hence

$$
\mu_{\beta_{i}}(\mathscr{A}) \leq \mu_{\beta_{i}}(\mathscr{F} \otimes \mathscr{G}) \quad \text { for all } i \in \mathbb{N} .
$$

Using continuity of intersection numbers, we may pass to the limit and obtain the desired inequality for $\mathscr{A}$, thus finishing the proof of Theorem 4.2.

\section{BOGOMOLOV-GIESEKER INEQUALITIES}

We first prove a Bogomolov-Gieseker inequality for locally free sheaves on a smooth projective surface which are semistable with respect to movable classes. This is a minor generalisation of [Miy87, 4.7], which considers rational classes only.

Theorem 5.1 (Bogomolov-Gieseker Inequality). Let X be a smooth, projective surface, let $\mathscr{E}$ be a torsion-free sheaf of $\mathscr{O}_{X}$-modules, of rank $r$. If $\mathscr{E}$ is semistable with respect to a movable curve class $\alpha \in \operatorname{Mov}(X) \backslash\{0\}$, then

$$
\Delta(\mathscr{E}):=2 r \cdot c_{2}(\mathscr{E})-(r-1) \cdot c_{1}^{2}(\mathscr{E}) \geq 0
$$

If $\mathscr{E}$ is not locally free, then Inequality (5.1.1) is strict.

Remark 5.2. If the sheaf $\mathscr{E}$ in Theorem 5.1 is even $\alpha$-stable, then this theorem can be deduced from [Miy87, 4.7], using the results of Section 3.

5.1. Preparation for the proof of Theorem 5.1. If $X$ is any normal projective variety, if $A$ is any Cartier divisor on $X$ and $i \in \mathbb{N}$ any number, recall that the function $m \mapsto h^{i}\left(X, \mathscr{O}_{X}(m \cdot A)\right)$ grows asymptotically at most like $m^{\operatorname{dim} X}$. To prepare for the proof of Theorem 5.1, we recall the following standard generalization, cf. [MP97, Part I, Lecture III, Prop. 3.3]. 
Lemma 5.3. Let $X$ be any projective variety, let $A, B, C \in \operatorname{Div}(X)$ be any three Cartierdivisors and $i \in \mathbb{N}$ be any number. If $h^{i}\left(X, \mathscr{O}_{X}(m \cdot A+B)\right)$ grows asymptotically at least like $m^{\operatorname{dim} X}$, then $h^{i}\left(X, \mathscr{O}_{X}(m \cdot A+B+C)\right)$ has the same asymptotic growth rate,

$$
h^{i}\left(X, \mathscr{O}_{X}(m \cdot A+B)\right) \sim h^{i}\left(X, \mathscr{O}_{X}(m \cdot A+B+C)\right) .
$$

In particular, taking $C=-B$ it follows that

$$
m^{\operatorname{dim} X} \lesssim h^{i}\left(X, \mathscr{O}_{X}(m \cdot A+B)\right) \sim h^{i}\left(X, \mathscr{O}_{X}(m \cdot A)\right) \lesssim m^{\operatorname{dim} X},
$$

so that equality of growth rates holds.

Proof. We prove Equation (5.3.1) using induction on the dimension of $X$. If $\operatorname{dim} X=0$, there is nothing to show. Let us therefore assume that $\operatorname{dim} X>0$, and that the claim was shown for all varieties of smaller dimension. Now, if $P \subset X$ is any prime Cartier divisor, consider the ideal sheaf sequence

$$
\left.0 \rightarrow \mathscr{O}_{X}(m A+B) \rightarrow \mathscr{O}_{X}(m A+B+P) \rightarrow \mathscr{O}_{X}(m A+B+P)\right|_{P} \rightarrow 0 .
$$

The following excerpt of the long exact cohomology sequence,

$$
\begin{aligned}
\overbrace{H^{i-1}\left(P,\left.\mathscr{O}_{X}(m A+B+P)\right|_{P}\right)}^{\lesssim m^{\operatorname{dim} X-1}} \rightarrow H^{i}\left(\mathscr{O}_{X}(m A+B)\right) \rightarrow \\
\rightarrow H^{i}\left(\mathscr{O}_{X}(m A+B+P)\right) \rightarrow \underbrace{H^{i}\left(P,\left.\mathscr{O}_{X}(m A+B+P)\right|_{P}\right)}_{\lesssim m^{\mathrm{dim} X-1}},
\end{aligned}
$$

will then show that we have equality of asymptotic growth rates,

$$
h^{i}\left(X, \mathscr{O}_{X}(m \cdot A+B)\right) \sim h^{i}\left(X, \mathscr{O}_{X}(m \cdot A+B+P)\right) .
$$

The same line of argument, using the sequence

$$
\left.0 \rightarrow \mathscr{O}_{X}(m A+B-P) \rightarrow \mathscr{O}_{X}(m A+B) \rightarrow \mathscr{O}_{X}(m A+B)\right|_{P} \rightarrow 0,
$$

yields an analogous equality,

$$
h^{i}\left(X, \mathscr{O}_{X}(m \cdot A+B)\right) \sim h^{i}\left(X, \mathscr{O}_{X}(m \cdot A+B-P)\right) .
$$

In essence, we have shown that the asymptotic growth rate does not change when adding or subtracting prime divisors. Write $C$ as a difference of two very ample prime divisors to conclude.

5.2. Proof of Theorem 5.1. The proof roughly follows the line of argument given in [Miy87, 4.3] or [MP97, Part I, Lecture III, 3.9].

Step 1: Setup. We argue by contradiction. To be precise, we assume that the following holds.

Assumption 5.4. There exists a smooth, projective surface $X$, a movable class $\alpha \in$ $\operatorname{Mov}(X) \backslash\{0\}$ and an $\alpha$-semistable, torsion-free sheaf $\mathscr{E}$ such that $\Delta(\mathscr{E})<0$.

These assumptions can be simplified. Recall from [HL10, Sect. 3.4] that

$$
\Delta(\mathscr{E}) \geq \Delta\left(\mathscr{E}^{* *}\right) \geq \Delta\left(\mathscr{E}^{*} \otimes \mathscr{E}^{* *}\right),
$$

and that the first inequality is strict if $\mathscr{E}$ is not locally free. Since $\mathscr{E}^{*} \otimes \mathscr{E}^{* *}$ is likewise $\alpha$-semistable by Theorem 4.2 , we are free to replace $\mathscr{E}$ by $\mathscr{E}^{*} \otimes \mathscr{E}^{* *}$ throughout the argument. Recalling that reflexive sheaves are locally free in codimension two, this amounts to assuming the following.

Assumption w.l.o.g. 5.5. The sheaf $\mathscr{E}$ is locally free and has trivial determinant, $\operatorname{det} \mathscr{E} \cong \mathscr{O}_{X}$. 
Step 2: Notation. Choose a Cartier divisor $H \in \operatorname{Div}(X)$ such that both $H$ and $H-$ $K_{X}$ are ample. Recalling that $\alpha$ is movable and therefore $\alpha^{2} \geq 0$, it follows from the Hodge index theorem that $H \cdot \alpha>0$. The same holds for the intersection with $H-K_{X}$, and we obtain the following inequalities that we note for future reference,

$$
\mu_{\alpha}\left(\mathscr{O}_{X}(H)\right)>0 \text { and } \mu_{\alpha}\left(\mathscr{O}_{X}\left(H-K_{X}\right)\right)>0 .
$$

Set $Y:=\mathbb{P}(\mathscr{E})$, and denote the bundle map by $\pi: Y \rightarrow X$. Choose a divisor $\mathbb{1} \in \operatorname{Div}(Y)$ such that $\mathscr{O}_{Y}(\mathbb{1}) \cong \mathscr{O}_{\mathbb{P}(\mathscr{E})}(1)$.

Step 3: Conclusion. Recall from the computations of [MP97, Part I, Lecture III, 3.6] that the assumption $\Delta(\mathscr{E})<0$ made in 5.4 implies that $\mathbb{1}^{\operatorname{dim} Y}>0$. By RiemannRoch,

$$
m^{\operatorname{dim} Y} \sim \chi\left(\mathscr{O}_{Y}(m \cdot \mathbb{1})\right)=\sum_{i=0}^{\operatorname{dim} Y}(-1)^{i} \cdot h^{i}\left(Y, \mathscr{O}_{Y}(m \cdot \mathbb{1})\right)
$$

The Leray spectral sequence implies that $h^{i}\left(Y, \mathscr{O}_{Y}(m \cdot \mathbb{1})\right)=h^{i}\left(X, \pi_{*} \mathscr{O}_{Y}(m \cdot \mathbb{1})\right)$, which vanishes for $i \geq 3$. Equation (5.5.2) therefore yields

$$
h^{0}\left(Y, \mathscr{O}_{Y}(m \cdot \mathbb{1})\right) \sim m^{\operatorname{dim} Y} \text { or } h^{2}\left(Y, \mathscr{O}_{Y}(m \cdot \mathbb{1})\right) \sim m^{\operatorname{dim} Y} .
$$

The subsequent Steps 5 and 6 will show that neither of these two possibilities is realised in our setup finishing the proof.

Step 5: Growth rate of $h^{0}(\cdots)$. Assume that $h^{0}\left(Y, \mathscr{O}_{Y}(m \cdot \mathbb{1})\right)$ grows asymptotically like $m^{\operatorname{dim} Y}$. Combining the results obtains so far, this implies the following.

$$
\begin{array}{rlr}
m^{\operatorname{dim} Y} & \sim h^{0}\left(Y, \mathscr{O}_{Y}\left(m \cdot \mathbb{1}-\pi^{*} H\right)\right) & \text { Lemma } 5.3 \\
& =h^{0}\left(X, \pi_{*} \mathscr{O}_{Y}(m) \otimes \mathscr{O}_{X}(-H)\right) & \\
& =\operatorname{dim}_{C} \operatorname{Hom}_{X}\left(\mathscr{O}_{X}(H), \operatorname{Sym}^{m} \mathscr{E}\right) . &
\end{array}
$$

This is absurd. In fact, we have seen in (5.5.1) that the $\alpha$-slope of the invertible sheaf $\mathscr{O}_{X}(H)$ is positive. On the other hand, recall from Assumption 5.5 that $\operatorname{Sym}^{m} \mathscr{E}$ has vanishing first Chern class. It is thus $\alpha$-semistable, with $\mu_{\alpha}\left(\operatorname{Sym}^{m} \mathscr{E}\right)=0$, by Corollary 4.3. As we have seen in Corollary 2.18, any morphism $\mathscr{O}_{X}(H) \rightarrow$ $\operatorname{Sym}^{m} \mathscr{E}$ is therefore zero. It follows that $h^{0}\left(Y, \mathscr{O}_{Y}(m \cdot \mathbb{1})\right)$ cannot grow like $m^{\operatorname{dim} Y}$.

Step 6: Growth rate of $h^{2}(\cdots)$. We repeat the argument of Step 5 with minor variations. Assuming that $h^{2}\left(Y, \mathscr{O}_{Y}(m \cdot \mathbb{1})\right)$ grows like $m^{\operatorname{dim} Y}$, we obtain, using again Lemma 5.3

$$
\begin{aligned}
m^{\operatorname{dim} Y} & \sim h^{2}\left(Y, \mathscr{O}_{Y}\left(m \cdot \mathbb{1}+\pi^{*} H\right)\right) & & \text { Lemma } 5.3 \\
& =h^{2}\left(X, \pi_{*} \mathscr{O}_{Y}(m) \otimes \mathscr{O}_{X}(H)\right) & & \text { Leray spectral sequence } \\
& =h^{0}\left(X, \operatorname{Sym}^{m} \mathscr{E}^{*} \otimes \mathscr{O}_{X}\left(-\left(H-K_{X}\right)\right)\right) & & \text { Serre duality } \\
& =\operatorname{dim}_{\mathbb{C}} \operatorname{Hom}_{X}\left(\mathscr{O}_{X}\left(H-K_{X}\right), \operatorname{Sym}^{m} \mathscr{E}^{*}\right) . & &
\end{aligned}
$$

As before, recall from (5.5.1) that the $\alpha$-slope of the invertible sheaf $\mathscr{O}_{X}\left(H-K_{X}\right)$ is positive and observe that $\operatorname{Sym}^{m} \mathscr{E}^{*}$ is $\alpha$-semistable with slope $\mu_{\alpha}\left(\operatorname{Sym}^{m} \mathscr{E}^{*}\right)=0$. It follows that $h^{2}\left(Y, \mathscr{O}_{Y}(m \cdot \mathbb{1})\right)$ cannot grow like $m^{\operatorname{dim} Y}$. 


\section{FLATNESS CRITERIA}

6.1. Flatness. In this section we discuss various flatness results. The relevant notion is the following.

Definition 6.1 (Flat and projectively flat sheaves). If $Y$ is any algebraic variety, and $\mathscr{G}$ is any locally free, analytic sheaf of rank $r$ on the underlying complex space $Y^{a n}$, we call $\mathscr{G}$ flat if it is defined by a representation

$$
\pi_{1}\left(Y^{a n}\right) \rightarrow \mathrm{Gl}(r, \mathbb{C})
$$

of the topological fundamental group $\pi_{1}\left(Y^{a n}\right)$. A locally free, algebraic sheaf on $Y$ is called flat if and only if the associated analytic sheaf is flat.

In a similar vein, call $\mathscr{G}$ projectively flat if $\mathbb{P}(\mathscr{G})$ is given by a representation $\pi_{1}\left(Y^{a n}\right) \rightarrow \operatorname{PGl}(r, \mathbb{C})$.

We refer the reader to [GKP13] for a discussion of flat sheaves on algebraic varieties. Projective flatness is discussed in [JR13] and in the references quoted there.

6.1.1. Flatness for sheaves on surfaces. Simpson proved in [Sim92] an important flatness criterion for semistable locally free sheaves. In fact, let $X$ be a projective manifold $X$ of dimension $n$ and let $\mathscr{E}$ be a locally free sheaf that is $H$-semistable for some ample divisor $H$. Suppose further that

$$
c_{1}(\mathscr{E}) \cdot H^{n-1}=\left(c_{1}(\mathscr{E})^{2}-c_{2}(\mathscr{E})\right) \cdot H^{n-2}=0 .
$$

Then, $\mathscr{E}$ is flat. We first generalise this in case $\operatorname{dim} X=2$, replacing $H$ by a movable class on the surface.

Theorem 6.2 (Criterion for flatness). Let $X$ be a smooth, projective surface and $\alpha \in$ $\operatorname{Mov}(X)$ a movable class. Let $\mathscr{E}$ be any torsion-free coherent sheaf on $X$ with

$$
c_{1}(\mathscr{E}) \cdot \alpha=c_{1}(\mathscr{E})^{2}-c_{2}(\mathscr{E})=0 .
$$

If $\mathscr{E}$ is $\alpha$-semistable and $\alpha^{2}>0$, then $\mathscr{E}$ is a locally free, flat sheaf.

Remark 6.3. The assumptions made in Theorem 6.2 are necessary. For an example where $\mathscr{E}$ is $\alpha$-semistable, where $\alpha^{2}=0$ and where $\mathscr{E}$ is not flat, let $\pi: X \rightarrow Y$ be any ruled surface, let $F$ be any fibre and set $\alpha:=[F]$ and $\mathscr{E}:=\mathscr{O}_{X}(F) \oplus \mathscr{O}_{X}(-F)$

Theorem 6.4 (Criterion for projective flatness). Let $X$ be a smooth, projective surface and $\alpha \in \operatorname{Mov}(X) \backslash\{0\}$ be a movable class. Let $\mathscr{E}$ be any $\alpha$-stable, torsion-free coherent sheaf on $X$ of rank $r$. If equality holds in the Bogomolov-Gieseker inequality,

$$
2 r \cdot c_{2}(\mathscr{E})=(r-1) \cdot c_{1}(\mathscr{E})^{2},
$$

then $\mathscr{E}$ is a projectively flat, locally free sheaf that is semistable with respect to every $\beta \in$ $\operatorname{Mov}(X)$. If we assume in addition that $c_{1}(\mathscr{E}) \cdot \alpha=c_{1}(\mathscr{E})^{2}=0$, i.e., we assume that (6.2.1) holds, then either $\mathscr{E}$ or $\mathscr{E}^{*}$ is nef.

6.1.2. Flatness in higher dimensions. We use the flatness criterion of Theorem 6.2 to generalise Theorem 1.19 from [GKP13].

Theorem 6.5 (Flatness criterion). Let X be a normal, projective, Q-factorial variety of dimension $n$ with only canonical singularities. Let $\mathscr{E}$ be a reflexive sheaf on $X$ and $H \in \operatorname{Div}(X)$ an ample divisor. Suppose that $\mathscr{E}$ is $H$-semistable and that there exists a desingularisation $\pi: \widetilde{X} \rightarrow X$ such that the following two equalities hold,

$$
\begin{aligned}
& 0=c_{1}(\mathscr{E}) \cdot H^{n-1} \\
& 0=c_{1}\left(\pi^{[*]} \mathscr{E}\right)^{2} \cdot\left(\pi^{*} H\right)^{n-2}-c_{2}\left(\pi^{[*]} \mathscr{E}\right) \cdot\left(\pi^{*} H\right)^{n-2} .
\end{aligned}
$$


Then, there exists a quasi-étale morphism $\gamma: \widehat{X} \rightarrow X$ such that $\gamma^{[*]} \mathscr{E}$ is a locally free, flat sheaf on $\widehat{X}$.

Remark 6.6. Notice that condition (6.5.2) does not depend on the choice of $\pi$, see $[\mathrm{GKP} 13,4.4]$ for the argument. It is essential that two desingularizations can be dominated by a third one.

Remark 6.7. Theorem 6.5 holds if $X$ has only klt singularities provided we knew that $\pi_{1}(X) \simeq \pi_{1}\left(X^{\circ}\right)$, where $X^{\circ}$ is the locus of quotient singularities.

Remark 6.8. One might ask whether Theorem 6.5 holds in greater generality, that is, for a larger class of singular spaces. What we actually needed is the following.

(6.8.1) A general complete intersection surface $S \subset X$ has only rational singularities.

(6.8.2) The following Lefschetz-type statement holds true: there exists a closed set $A \subset X$ of codimension at least three such that $\pi_{1}(S) \simeq \pi_{1}(X \backslash A)$.

(6.8.3) There exists a quasi-étale cover $\widetilde{X} \rightarrow X$ such that $\widehat{\pi}_{1}\left(\widetilde{X}_{\text {reg }}\right) \simeq \widehat{\pi}_{1}(\widetilde{X})$.

Corollary 6.9. Let $X$ be a normal $Q$-factorial projective variety with only canonical singularities. Let $H \neq 0$ be any nef divisor on $X$ and $\mathscr{E}=\bigoplus \mathscr{E}_{i}$ a reflexive sheaf on $X$ whose direct summands are $H$-stable. If $c_{1}\left(\mathscr{E}_{i}\right)=0$ for all $i$ and if there exists a desingularisation $\pi: \widetilde{X} \rightarrow X$ such that $\pi^{[*]} \mathscr{E}$ is locally free and $c_{2}\left(\pi^{[*]} \mathscr{E}\right)=0$, then there exists a quasi-étale morphism $\gamma: \widehat{X} \rightarrow X$ such that $\gamma^{[*]} \mathscr{E}$ is a flat, locally free sheaf on $\widehat{X}$.

Proof. If $H_{0}$ is any ample divisor, it will follow from Theorem 3.4 that all direct summands $\mathscr{E}_{i}$ are stable with respect to $\left(H+\varepsilon \cdot H_{0}\right)$, for all sufficiently small positive $\varepsilon$. More is true. Since $c_{1}\left(\mathscr{E}_{i}\right)=0$ the bundle $\mathscr{E}$ is polystable with respect to $\left(H+\varepsilon \cdot H_{0}\right)$, and in particular semistable. Now apply Theorem 6.5.

6.2. Preparations. The proof of Theorem 6.2 makes use of the following lemmas, which might be of independent interest.

Lemma 6.10. Let $X$ be a smooth, projective surface and $\Delta \in N^{1}(X)_{Q}$ a rational divisor class. Then, either there exists an integral, ample divisor $H$ such that $H \cdot \Delta=0$, or either $\Delta$ or $-\Delta$ is pseudo-effective.

Proof. Since $X$ is a surface, divisors and curves coincide, and the interior of the movable cone equals the ample cone. Since $\Delta$ is a rational class by assumption, the hyperplane

$$
\Delta^{\perp}:=\left\{\alpha \in N_{1}(X)_{\mathbb{R}} \mid \alpha \cdot \Delta=0\right\}
$$

is likewise rational, and rational points are dense there. If $\Delta^{\perp}$ intersects the interior of the movable cone at all, the intersection will thus contain a rational point. In other words, there will be integral ample divisors $H$ with $H \cdot \Delta=0$.

Assuming that no such divisor $H$ exists therefore amounts to assuming that the function $\bullet \cdot \Delta$ does not have any zeros in $\operatorname{Mov}(X)^{\circ}$ and is either strictly positive or strictly negative there. As seen in Remark 2.3 on page 4 , this means that either $\Delta$ or $-\Delta$ is pseudo-effective.

Lemma 6.11. Let $X$ be a smooth, projective surface and $\Delta \in N^{1}(X)_{Q}$ a rational, pseudo-effective divisor class with $\Delta^{2}=0$. Assume that there exists a movable class $\alpha \in \operatorname{Mov}(X) \backslash\{0\}$ such that $\alpha \cdot \Delta=0$. Then, $\Delta$ is nef.

Proof. Consider the Zariski decomposition, $\Delta=[P]+[N]$, where $P$ and $N$ are $Q$ divisors, where $P$ is nef, $N=\sum a_{i} N_{i}$ is effective, $N^{2}<0$ unless $N=0$, and finally $P \cdot N_{i}=0$ for all $i$.

Observe that the movable (=nef) class $\alpha$ intersects $P$ and $N$ non-negatively. The assumption that $\alpha \cdot \Delta=0$ therefore gives that $\alpha \cdot P=\alpha \cdot N=0$. Since $\alpha \neq 0$ and 
$\alpha^{2} \geq 0$, the Hodge index theorem thus implies that the non-negative number $P^{2}$ is actually zero. Since $0=\Delta^{2}=P^{2}+N^{2}$, we obtain that $N^{2}=0$, and hence that $N=0$. It follows that $\Delta=[P]$ is nef.

6.3. Proof of Theorem 6.2. Using the assumption that $\alpha^{2}>0$, the Hodge index theorem asserts that $c_{1}(\mathscr{E})^{2} \leq 0$. Thus, the assumption $c_{1}(\mathscr{E})^{2}=c_{2}(\mathscr{E})$, combined with the Bogomolov-Gieseker inequality of Theorem 5.1 yields $c_{1}(\mathscr{E})^{2}=c_{2}(\mathscr{E})=$ 0 , and taking into account the further assumption made in (6.2.1) the Hodge index theorem hence asserts that $c_{1}(\mathscr{E})=0$. In particular, equality holds in the Bogomolov-Gieseker inequality of Theorem 5.1, and $\mathscr{E}$ is therefore locally free.

Step 1: Proof in case where $\mathscr{E}$ is stable. If $\mathscr{E}$ is $\alpha$-stable, Theorem 3.4 and Remark 3.5 allow to find a rational point $h^{\prime} \in[\mathscr{E}]^{\perp} \cap \operatorname{Mov}(X)^{\circ} \cap \operatorname{Stab}(\mathscr{E})$. If $H^{\prime}$ is a divisor with class $h^{\prime},\left[H^{\prime}\right]=h^{\prime}$, then $H^{\prime}$ is ample, and it follows from classical theorems that $\mathscr{E}$ is flat, e.g. see [Sim92, Cor. 3.10]. To apply Simpson's theorem, we view $\mathscr{E}$ as a Higgs bundle with zero Higgs field $\theta=0$ and notice that slope semi-stability in our sense implies semi-stability of Higgs bundles.

Step 2: Proof in general. Consider a minimal-length Jordan-Hölder filtration of $\mathscr{E}$, as discussed in Corollary 2.28,

$$
0=\mathscr{E}_{0} \subsetneq \mathscr{E}_{1} \subsetneq \cdots \subsetneq \mathscr{E}_{k}=\mathscr{E} .
$$

The proof proceeds by induction on the length of the filtration, denoted by $k$. If $k=1$, then $\mathscr{E}$ is $\alpha$-stable, and the assertion has been shown in Step 1. We will therefore assume for the remainder of the proof that $k>1$, and that the claim has already been shown for all $\alpha$-semistable bundles satisfying Equations (6.2.1) that admit a shorter Jordan-Hölder filtration.

For brevity of notation write $\mathscr{F}:=\mathscr{E}_{k-1}$ and $\mathscr{Q}:=\mathscr{E} / \mathscr{F}$. We obtain a sequence

$$
0 \rightarrow \mathscr{F} \rightarrow \mathscr{E} \rightarrow \mathscr{Q} \rightarrow 0,
$$

where $\mathscr{F}$ is $\alpha$-semistable, reflexive and therefore locally free, and where $\mathscr{Q}$ is $\alpha$ stable and torsion-free, and $c_{1}(\mathscr{F}) \cdot \alpha=c_{1}(\mathscr{Q}) \cdot \alpha=0$. As before, the Hodge index theorem will thus imply the following.

$$
c_{1}(\mathscr{F})^{2} \leq 0 \quad \text { and } \quad c_{1}(\mathscr{Q})^{2} \leq 0, \quad \text { each with equality iff } c_{1}(\cdot)=0 .
$$

We aim to show that the vanishing (6.2.1) holds for $\mathscr{F}$ and $\mathscr{Q}$. Since both $\mathscr{F}$ and $\mathscr{Q}$ admit Jordan-Hölder filtrations of length less than $k$, flatness of $\mathscr{F}$ and $\mathscr{Q}$ then follows from the induction hypothesis. The bundle $\mathscr{E}$ is then presented as an extension of two flat bundles and is therefore flat by [Sim92, 3.10]. $\mathscr{Q}$,

To this end, we use the following relations between the Chern classes of $\mathscr{F}$ and

$$
\begin{aligned}
& 0=c_{1}(\mathscr{E})^{2}=c_{1}(\mathscr{F})^{2}+c_{1}(\mathscr{Q})^{2}+2 \cdot c_{1}(\mathscr{F}) \cdot c_{1}(\mathscr{Q}) \\
& 0=c_{2}(\mathscr{E})=c_{2}(\mathscr{F})+c_{2}(\mathscr{Q})+c_{1}(\mathscr{F}) \cdot c_{1}(\mathscr{Q})
\end{aligned}
$$

These equations yield

$$
\begin{aligned}
\frac{1}{2} c_{1}(\mathscr{F})^{2}+\frac{1}{2} c_{1}(\mathscr{Q})^{2} & =c_{2}(\mathscr{F})+c_{2}(\mathscr{Q}) & \text { (6.12.2) and (6.12.3) } \\
& \geq \underbrace{\frac{\operatorname{rank} \mathscr{F}-1}{2 \operatorname{rank} \mathscr{F}}}_{\text {non-neg., }<1 / 2} \cdot c_{1}(\mathscr{F})^{2}+\underbrace{\frac{\operatorname{rank} \mathscr{Q}-1}{2 \operatorname{rank} \mathscr{Q}}}_{\text {non-neg., }<1 / 2} \cdot c_{1}(\mathscr{Q})^{2} & \text { BGI, Theorem 5.1. }
\end{aligned}
$$

Combined with (6.12.1), this shows that $c_{1}(\mathscr{F})^{2}=c_{1}(\mathscr{Q})^{2}=0$. Applying the Bogomolov-Gieseker Inequality, Theorem 5.1, once more to $\mathscr{F}$ and $\mathscr{Q}$, we see that $c_{2}(\mathscr{F}) \geq 0$ and $c_{2}(\mathscr{Q}) \geq 0$, and it follows from (6.12.3) that both numbers vanish. 
The induction hypothesis therefore applies to show that $\mathscr{F}$ and $\mathscr{Q}$ are flat. As noted above, this proves flatness of $\mathscr{E}$.

6.4. Proof of Theorem 6.4. Let $\mathscr{E}$ be any $\alpha$-stable, torsion-free sheaf on $X$ such that equality holds in the Bogomolov-Gieseker inequality. By Theorem 5.1, the sheaf $\mathscr{E}$ is then locally free.

Step 1: Proof of projective flatness. We have seen in Theorem 3.4 that $\mathscr{E}$ is stable also with respect to a suitable rational class $\beta$ that is contained in the interior of the movable cone. Since $X$ is a surface, the movable cone equals the nef cone, and its interior consists of ample classes. Projective flatness is now a consequence of a classical theorem, see [JR13, Thm. 1.1 and Prop. 1.1] for a discussion and for further references. This paper also asserts that $\mathscr{E}$ is semistable with respect to any ample class. Semistability with respect to all classes in $\operatorname{Mov}(X) \backslash\{0\}$ then follows from Remark 3.2, since $\operatorname{Mov}(X)$ is the closure of the ample cone.

Step 2: Proof of nefness. Assuming that the Equalities (6.2.1) hold, we aim to prove that $\mathscr{E}$ or $\mathscr{E}^{*}$ are nef, or equivalently, that $\operatorname{Sym}^{r}(\mathscr{E})$ or $\operatorname{Sym}^{r}\left(\mathscr{E}^{*}\right)$ are nef vector bundles, where $r:=\operatorname{rank} \mathscr{E}$, cf. [Laz04, Thm. 6.2.12]. Since both $\operatorname{Sym}^{r}(\mathscr{E}) \otimes \operatorname{det} \mathscr{E} *$ and its dual are nef by projective flatness, [JR13, Sect. 1.3], it suffices to show that either $\operatorname{det} \mathscr{E}$ or $\operatorname{det} \mathscr{E} *$ is nef.

To this end, observe that if there exists an integral, ample divisor $H$ such that $H \cdot c_{1}(\mathscr{E})=0$, then Theorem 3.4 allows to find a rational point $h^{\prime} \in c_{1}(\mathscr{E})^{\perp} \cap$ $\operatorname{Mov}(X)^{\circ} \cap \operatorname{Stab}(\mathscr{E})$. Any divisor $H^{\prime}$ with class $h^{\prime}$ is then ample, and it follows from Simpson's theorem that $\mathscr{E}$ is flat, [Sim92, Cor. 3.10]. The class $c_{1}(\mathscr{E})$ will then vanish, and we are done.

We will thus assume that no such divisor $H$ exists. One of the divisors $\operatorname{det} \mathscr{E}$ or $\operatorname{det} \mathscr{E}^{*}$ is then pseudo-effective by Lemma 6.10. Using the assumption that $c_{1}(\mathscr{E})$. $\alpha=c_{1}(\mathscr{E})^{2}=0$, nefness now follows from Lemma 6.11 .

6.5. Proof of Theorem 6.5. Our subsequent proof of Theorem 6.5 will use the following minimal generalisation of a result in [GKP13]. The proof is exactly the same as the one given there, and therefore omitted.

Lemma 6.13 (Iterated Bertini-type theorem for bounded families, cf. [GKP13, Cor. 5.6]). Let $X$ be a normal, projective variety of dimension $\operatorname{dim} X \geq 2$. Let $\mathscr{E}$ be a coherent, reflexive sheaf of $\mathscr{O}_{X}$-modules, and let $F$ be a bounded family of locally free sheaves. Given an ample line bundle $\mathscr{L} \in \operatorname{Pic}(X)$, a sufficiently increasing sequence $0 \ll m_{1} \ll m_{2} \ll \cdots \ll m_{k}$ and general elements $H_{i}$ of a basepoint-free linear system contained in $\left|\mathscr{L}^{\otimes m_{i}}\right|$ with associated complete intersection variety $S:=H_{1} \cap \cdots \cap H_{k}$, then the following holds for all sheaves $\mathscr{F} \in F$. The sheaf $\mathscr{F}$ is isomorphic to $\mathscr{E}$ if and only if $\left.\mathscr{F}\right|_{S}$ is isomorphic to $\left.\mathscr{E}\right|_{S}$.

Proof of Theorem 6.5. Let $\gamma: \widehat{X} \rightarrow X$ be the finite, quasi-étale cover guaranteed by [GKP13, Thm. 1.13], which has the property that any locally free, flat sheaf defined on $\widehat{X}_{\text {reg }}^{a n}$ extends to a locally free, flat sheaf on the whole of $\widehat{X}$.

We may assume $H$ to be sufficiently ample in the sense of Mehta-Ramanathan and so that Lemma 6.13 may be applied to the linear subsystem $\gamma^{*}|H|$ of $\left|\gamma^{*}(H)\right|$ without taking further multiples. Choose a general tuple of hypersurfaces $D_{1}, \ldots, D_{n-2} \in|H|$ and write

$$
S:=D_{1} \cap \cdots \cap D_{n-2} .
$$

We recall the classical fact, that in codimension two a variety with canonical singularities is locally in the Euclidean topology a product of a surface with an 
ADE singularity and of a smooth space of dimension $(\operatorname{dim} X-2)$, see for example [GKKP11, Prop. 9.3]. In particular, the set $Z \subset X$ where $X$ is not locally a complete intersection is small, $\operatorname{codim}_{X} Z \geq 3$. Set $X^{\circ}:=X \backslash Z$ and note that $S \subset X^{\circ}$.

Since $S$ is likewise canonical, it is normal with only ADE singularities. In particular, $S$ is $Q$-factorial. By Flenner's version of the Mehta-Ramanathan theorem, [Fle84, Thm. 1.2], the restriction $\left.\mathscr{E}\right|_{S}$ is semistable with respect to the ample class $\left.H\right|_{S}$.

Let $\widetilde{D}_{i}:=\pi^{*}\left(D_{i}\right) \in\left|\pi^{*} H\right|$ and set $\widetilde{S}:=\widetilde{D}_{1} \cap \cdots \cap \widetilde{D}_{n-2}$. Then $\widetilde{S}=\pi^{-1}(S)$ is a smooth surface contained in the smooth locus of $\widetilde{X}$. For convenience of notation, write

$$
g:=\left.\pi\right|_{S}, \quad \widetilde{E}:=g^{[*]}\left(\left.\mathscr{E}\right|_{S}\right) \quad \text { and } \quad \widetilde{H}:=g^{*}\left(\left.H\right|_{S}\right) .
$$

We have seen in Proposition 2.8 on page 5 that the pull-back sheaf $\widetilde{\mathscr{E}}$ is stable with respect to $\widetilde{H}$. Equations (6.5.1) and (6.5.2) then read as follows,

$$
0=c_{1}(\widetilde{\mathscr{E}}) \cdot \widetilde{H} \quad \text { and } \quad 0=c_{1}(\widetilde{\mathscr{E}})^{2}-c_{2}(\widetilde{\mathscr{E}}) .
$$

For the second equation, we observe that $\left.\pi^{[*]}(\mathscr{E})\right|_{\widetilde{S}}$ is reflexive and thus equals $\widetilde{\mathscr{E}}$. In particular, Theorem 6.2 implies that $\widetilde{E}$ is flat, hence given by a representation $\pi_{1}(\widetilde{S}) \rightarrow \mathrm{Gl}(r, \mathbb{C})$. Since $S$ has only canonical singularities, $\pi_{1}(\widetilde{S}) \cong \pi_{1}(S)$, and the induced representation $\pi_{1}(S) \rightarrow \operatorname{Gl}(r, \mathbb{C})$ defines a locally free flat sheaf $\mathscr{F}$ on $S$ such that $p^{*} \mathscr{F} \simeq \widetilde{\mathscr{E}}$. It follows that the sheaves $\mathscr{F}$ and $\left.\mathscr{E}\right|_{S}$ agree outside a finite set of $S$. Since $\left.\mathscr{E}\right|_{S}$ is reflexive, [GKP13, Proposition 5.2], the sheaves $\mathscr{F}$ and $\left.\mathscr{E}\right|_{S}$ are isomorphic. Since $X^{\circ}$ is a locally complete intersection variety, a Lefschetztype theorem of Goresky-MacPherson [GM88, II.1.2, Thm. on p. 153] asserts that the inclusion $\iota: S \rightarrow X^{\circ}$ induces an isomorphism

$$
\iota_{*}: \pi_{1}(S) \rightarrow \pi_{1}\left(X^{\circ}\right) \text {. }
$$

Thus, there exists a locally free, flat sheaf $\mathscr{G}$ on $X^{\circ}$ such that $\left.\left.\mathscr{G}\right|_{S} \cong \mathscr{E}\right|_{S}$. The observation that Zariski-open subsets with complement of codimension at least two inside a smooth variety have the same fundamental group as the entire variety together with the choice of $\widehat{X}$ implies that the pullback of $\mathscr{G}$ to $\gamma^{-1}\left(X^{\circ}\right) \cap \widehat{X}_{\text {reg }}$ extends to a locally free, flat sheaf $\widehat{\mathscr{G}}$ on the whole of $\widehat{X}$. Setting $\widehat{S}:=\gamma^{-1}(S)=$ $\gamma^{*} D_{1} \cap \ldots \cap \gamma^{*} D_{n-2}$, we note that

$$
\left.\left.\widehat{\mathscr{G}}\right|_{\widehat{S}} \cong\left(\left.\gamma\right|_{\widehat{S}}\right)^{*}\left(\left.\mathscr{E}\right|_{S}\right) \cong\left(\gamma^{[*]} \mathscr{E}\right)\right|_{\widehat{S}}
$$

As the set of all locally free, flat sheaves on $\widehat{X}$ whose rank is equal to rank $\mathscr{E}$ is bounded by [GKP13, Prop. 12.1], we may apply Lemma 6.13 above to conclude that $\gamma^{[*]} \mathscr{E}$ is isomorphic to $\widehat{\mathscr{G}}$, and hence locally free and flat, as claimed.

\section{CHARACTERISATION OF TORUS QUOTIENTS}

The main result of this section generalises a result for three-dimensional varieties of Shepherd-Barron and Wilson [SBW94, Cor. of Main Thm], and eliminates the a priori assumption on the codimension of the singular locus made in [GKP13, Thm. 1.16].

Theorem 7.1 (Characterisation of torus quotients). Let X be a normal Q-factorial projective variety of dimension $n$ with only canonical singularities and numerically trivial canonical bundle, $K_{X} \equiv 0$. Assume that there exists a desingularisation $\pi: \widetilde{X} \rightarrow X$ and an ample divisor $H \in \operatorname{Dix}(X)$ such that $c_{2}(\widetilde{X}) \cdot\left(\pi^{*} H\right)^{n-2}=0$. Then, $X$ is smooth in codimension two, there exists an Abelian variety $A$ and a quasi-étale morphism $\gamma: A \rightarrow$ $X$. 
Theorem 7.1 is shown below, on the facing page. It follows almost immediately from the following two lemmas.

Lemma 7.2. Let $\pi: \widetilde{S} \rightarrow S$ be a desingularisation of a normal, projective, Q-factorial surface $S$ with only rational singularities. Let $\alpha \in \operatorname{Mov}(S)$ be a movable class with $\alpha^{2}>0$ and let $\mathscr{E}$ be a torsion-free, $\left(\pi^{*} \alpha\right)$-semistable, coherent sheaf on $\widetilde{S}$ such that

$$
c_{1}(\mathscr{E}) \cdot\left(\pi^{*} \alpha\right)=c_{1}(\mathscr{E})^{2}-c_{2}(\mathscr{E})=0
$$

Then, $\pi_{*} \mathscr{E}$ is locally free and flat on $S$.

Proof. Write $r:=\operatorname{rank} \mathscr{E}$. It follows directly from Theorem 6.2 that $\mathscr{E}$ is flat, given by a representation $\rho: \pi_{1}(\widetilde{S}) \rightarrow G L_{r}(\mathbb{C})$. If $x \in S$ is any singular point with associated fibre $F:=\pi^{-1}(x)$, then $F$ is simply-connected and $\left.\mathscr{E}\right|_{F}$, which is given by a representation of $\pi_{1}(F)$, therefore trivial. Consequently, if $\mathscr{E}$ ' is the locally free, flat sheaf defined by $\rho \circ\left(\pi_{*}\right)^{-1}: \pi_{1}(S) \rightarrow G L_{r}(\mathbb{C})$, we have $\mathscr{E}=\pi^{*} \mathscr{E}$ and $\mathscr{E}^{\prime}=\pi_{*} \mathscr{E}$.

Lemma 7.3. Let $X$ be a normal, projective variety of dimension $n \geq 2$ with numerically trivial canonical class, $K_{X} \equiv 0$, that has at most canonical singularities. Let $\pi: \widetilde{X} \rightarrow X$ be any resolution of singularities and $H$ an ample divisor on $X$. If $c_{2}(\widetilde{X}) \cdot\left(\pi^{*} H\right)^{n-2}=0$, then $X$ is smooth in codimension two.

Proof. We may assume without loss of generality that $\mathscr{H}:=\mathscr{O}_{X}(H)$ is very ample. Choose a general $(n-2)$-tuple of elements $H_{i} \in|\mathscr{H}|$ and consider the associated complete intersection surface $S:=\cap_{i} H_{i} \subseteq X$, which has at worst canonical singularities. We aim to show that $X$ is smooth near $S$. Write

$$
\mathscr{N}_{S / X}:=\left(\left.\mathscr{H}\right|_{S}\right)^{\oplus n-2}, \quad \widetilde{S}:=\pi^{-1}(S) \text { and } \pi_{S}:=\left.\pi\right|_{\widetilde{S}} .
$$

Since $X$ has canonical singularities, we may write $K_{\widetilde{X}}=\pi^{*} K_{X}+D \equiv D$, where $D$ is effective and $\pi$-exceptional. We obtain the following equalities of intersection numbers,

$$
\left[\left.K_{\widetilde{X}}\right|_{\widetilde{S}}\right] \cdot\left[\mathscr{N}_{\widetilde{S} / \widetilde{X}}^{*}\right]=\left[\left.D\right|_{\widetilde{S}}\right] \cdot\left[\mathscr{N}_{\widetilde{S} / \widetilde{X}}^{*}\right]=-\left[\left.D\right|_{\widetilde{S}}\right] \cdot\left[\pi_{S}^{*} \mathscr{N}_{S / X}\right]=0
$$

and hence

$$
\left[K_{\widetilde{S}}\right] \cdot\left[\mathscr{N}_{\widetilde{S} / \widetilde{X}}^{*}\right]=\left(\left[\left.K_{\widetilde{X}}\right|_{\widetilde{S}}\right]+\left[\mathscr{N}_{\widetilde{S} / \widetilde{X}}\right]\right) \cdot\left[\mathscr{N}_{\widetilde{S} / \widetilde{X}}^{*}\right]=-\left[\mathscr{N}_{\widetilde{S} / \widetilde{X}}\right]^{2}
$$

The normal bundle sequence for $\widetilde{S}$ in $\widetilde{X}$ expresses the relevant second Chern class as follows,

$$
\begin{aligned}
0=c_{2}\left(\left.\Omega_{\widetilde{X}}^{1}\right|_{\widetilde{S}}\right) & =c_{2}\left(\mathscr{N}_{\widetilde{S} / \widetilde{X}}^{*}\right)+c_{2}\left(\Omega_{\widetilde{S}}^{1}\right)+\left[K_{\widetilde{S}}\right] \cdot\left[\mathscr{N}_{\widetilde{S} / \widetilde{X}}^{*}\right] \\
& =c_{2}\left(\mathscr{N}_{\widetilde{S} / \widetilde{X}}^{*}\right)+\chi_{\text {top }}(\widetilde{S})-\left[\mathscr{N}_{\widetilde{S} / \widetilde{X}}\right]^{2} \quad \text { by (7.3.1). }
\end{aligned}
$$

We now compare $\pi$ to a Q-factorial terminalisation $\rho: \widehat{X} \rightarrow X$ of $X$, which exists by [BCHM10, Cor. 1.4.3]. As the name suggests, $\widehat{X}$ is $\mathbb{Q}$-factorial and has at most terminal singularities. Moreover, as $X$ has canonical singularities, $\rho$ is crepant, and

$$
K_{\widehat{X}} \sim_{Q} \rho^{*} K_{X} \equiv 0
$$

Write $\widehat{S}:=\rho^{-1}(S)$ and $\rho_{S}:=\left.\rho\right|_{\widehat{S}}$. Since varieties with terminal singularities are smooth in codimension two, the surface $\widehat{S}$ is smooth, and entirely contained in the smooth locus of $\widehat{X}$. As above, compute

$$
\begin{aligned}
c_{2}\left(\left.\Omega_{\widehat{X}}^{1}\right|_{\widehat{S}}\right) & =c_{2}\left(\mathscr{N}_{\widehat{S} / \widehat{X}}^{*}\right)+c_{2}\left(\Omega_{\widehat{S}}^{1}\right)+\left[K_{\widehat{S}}\right] \cdot\left[\mathscr{N}_{\widehat{S} / \widehat{X}}\right] \\
& =c_{2}\left(\mathscr{N}_{\widehat{S} / \widehat{X}}^{*}\right)+\chi_{\text {top }}(\widehat{S})-\left[\mathscr{N}_{\widehat{S} / \widehat{X}}\right]^{2} .
\end{aligned}
$$


It follows from adjunction and from the Q-linear equivalence $K_{\widehat{X}} \sim_{\mathbb{Q}} \rho^{*} K_{X}$ that $K_{\widehat{S}} \sim_{Q}\left(\left.\rho\right|_{\widehat{S}}\right)^{*} K_{S}$, so that $\widehat{S}$ is in fact the minimal resolution of $S$. Consequently, there exists a birational $S$-morphism $\beta: \widetilde{S} \rightarrow \widehat{S}$, which is a sequence of blowingdown $(-1)$-curves. This has two consequences. First, there is an inequality of topological Euler characteristics, $\chi_{\text {top }}(\widehat{S}) \leq \chi_{\text {top }}(\widetilde{S})$. Secondly, we see that

$$
\mathscr{N}_{\widetilde{S} / \widetilde{X}}=\pi_{S}^{*} \mathscr{N}_{S / X}=\beta^{*} \rho_{S}^{*} \mathscr{N}_{S / X}=\beta^{*} \mathscr{N}_{\widehat{S} / \widehat{X}^{\prime}}
$$

so that the Chern numbers of the normal bundles agree, $c_{2}\left(\mathscr{N}_{\tilde{S} / \tilde{X}}\right)=c_{2}\left(\mathscr{N}_{\widehat{S} / \widehat{X}}\right)$ and $\left[\mathscr{N}_{\widetilde{S} / \widetilde{X}}\right]^{2}=\left[\mathscr{N}_{\widehat{S} / \widehat{X}}\right]^{2}$. Comparing (7.3.2) and (7.3.4), we thus see that $c_{2}\left(\left.\Omega_{\widehat{X}}^{1}\right|_{\widehat{S}}\right) \leq 0$.

To end the argument, let $\check{X} \rightarrow \widehat{X}$ be a strong resolution of singularities, with induced map $r: \breve{X} \rightarrow X$, surface $\breve{S}:=r^{-1}(S) \subseteq \check{X}$ and restriction $r_{S}:=\left.r\right|_{\breve{S}}$. Since $\check{X}$ and $\widehat{X}$ are isomorphic along $\widehat{S}$, we have $c_{2}\left(\left.\Omega_{\breve{X}}^{1}\right|_{\breve{S}}\right) \leq 0$, and Miyaoka's Chern class inequalities, [Miy87, Thm. 1.1], assert that in fact equality holds, $c_{2}\left(\left.\Omega_{\breve{X}}^{1}\right|_{\breve{S}}\right)=0$.

To sum up, we have seen in (7.3.3) that

$$
c_{1}\left(\left.\Omega_{\check{X}}^{1}\right|_{\breve{S}}\right)=\left[\left.K_{\check{X}}\right|_{\breve{S}}\right]=\left[\left.K_{\widehat{X}}\right|_{\widehat{S}}\right] \equiv 0
$$

and thus

$$
\left.c_{1}\left(\left.\Omega_{\breve{X}}^{1}\right|_{\breve{S}}\right) \cdot\left(r^{*} H\right)\right|_{\check{S}}=c_{1}\left(\left.\Omega_{\breve{X}}^{1}\right|_{\breve{S}}\right)^{2}-c_{2}\left(\left.\Omega_{\breve{X}}^{1}\right|_{\breve{S}}\right)=0 .
$$

Lemma 7.2 thus applies to show that $\left(r_{S}\right)_{*}\left(\left.\Omega_{\breve{X}}^{1}\right|_{\breve{S}}\right)$ is locally free and flat. Set $\Omega_{X}^{[1]}:=\left(\Omega_{X}^{1}\right)^{* *}$. Since $\left.\Omega_{X}^{[1]}\right|_{S}$ is reflexive by [GKP13, Prop. 5.2], it necessarily agrees with $\left(r_{S}\right)_{*}\left(\left.\Omega_{\check{X}}^{1}\right|_{\breve{S}}\right)$, showing that both $\Omega_{X}^{[1]}$ and $\mathscr{T}_{X}=\left(\Omega_{X}^{[1]}\right)^{*}$ are locally free near $S$. The Lipman-Zariski theorem for canonical spaces, [GKKP11, Thm. 6.1] or [Dru14, Thm. 3.8], thus applies, showing that $X$ is smooth near $S$.

Proof of Theorem 7.1. Lemma 7.3 asserts that $X$ is smooth in codimension two. Under this additional assumption, Theorem 7.1 has been shown in [GKP13, Thm. 1.16].

\section{REFERENCES}

[BCHM10] Caucher Birkar, Paolo Cascini, Christopher D. Hacon, and James McKernan. Existence of minimal models for varieties of log general type. Journal of the AMS, 23:405-468, 2010. DOI:10.1090/S0894-0347-09-00649-3. $\uparrow 22$

[BDPP13] Sébastien Boucksom, Jean-Pierre Demailly, Mihai Păun, and Thomas Peternell. The pseudo-effective cone of a compact Kähler manifold and varieties of negative Kodaira dimension. J. Algebraic Geom., 22(2):201-248, 2013. arXiv:math/0405285. $\uparrow 4$

[CP11] Frédéric Campana and Thomas Peternell. Geometric stability of the cotangent bundle and the universal cover of a projective manifold. Bull. Soc. Math. France, 139(1):41-74, 2011. $\uparrow 2$, 14

[Dru14] Stéphane Druel. The Zariski-Lipman conjecture for log canonical spaces. Bull. London Math. Soc., 46(4):827-835, 2014. $\uparrow 23$

[Fle84] Hubert Flenner. Restrictions of semistable bundles on projective varieties. Comment. Math. Helv., 59(4):635-650, 1984. $\uparrow 6,21$

[GKKP11] Daniel Greb, Stefan Kebekus, Sándor J. Kovács, and Thomas Peternell. Differential forms on log canonical spaces. Inst. Hautes Études Sci. Publ. Math., 114(1):87-169, November 2011. DOI:10.1007/s10240-011-0036-0 An extended version with additional graphics is available as arXiv:1003.2913. $\uparrow 21,23$

[GKP13] Daniel Greb, Stefan Kebekus, and Thomas Peternell. Étale fundamental groups of Kawamata log terminal spaces, flat sheaves, and quotients of Abelian varieties. Preprint arXiv:1307.5718, July 2013. $\uparrow 2,3,6,17,18,20,21,23$

[GKP14] Daniel Greb, Stefan Kebekus, and Thomas Peternell. Reflexive differential forms on singular spaces. Geometry and cohomology. J. Reine Angew. Math., 697:57-89, 2014. DOI:10.1515/crelle-2012-0097. Preprint arXiv:1202.3243. $\uparrow 2,4,7$ 
[GM88] Mark Goresky and Robert D. MacPherson. Stratified Morse theory, volume 14 of Ergebnisse der Mathematik und ihrer Grenzgebiete (3) [Results in Mathematics and Related Areas (3)]. Springer-Verlag, Berlin, 1988. $\uparrow 21$

[GRT14] Daniel Greb, Julius Ross, and Matei Toma. Variation of Gieseker Moduli Spaces via Quiver GIT, I. Preprint arXiv:1409.7564, September 2014. $\uparrow 9$

[GT13] Daniel Greb and Matei Toma. Compact moduli spaces for slope-semistable sheaves. Preprint arXiv:1303.2480, September 2013. $\uparrow 2$

[HL10] Daniel Huybrechts and Manfred Lehn. The geometry of moduli spaces of sheaves. Cambridge Mathematical Library. Cambridge University Press, Cambridge, second edition, 2010. $\uparrow 2$, 8,15

[JR13] Priska Jahnke and Ivo Radloff. Semistability of restricted tangent bundles and a question of I. Biswas. Internat. J. Math., 24(1):1250122, 15, 2013. $\uparrow 17,20$

[KM98] János Kollár and Shigefumi Mori. Birational geometry of algebraic varieties, volume 134 of Cambridge Tracts in Mathematics. Cambridge University Press, Cambridge, 1998. $\uparrow 6$

[Ko196] János Kollár. Rational curves on algebraic varieties, volume 32 of Ergebnisse der Mathematik und ihrer Grenzgebiete. 3. Folge. A Series of Modern Surveys in Mathematics. Springer-Verlag, Berlin, 1996. $\uparrow 3,4$

[Laz04] Robert Lazarsfeld. Positivity in algebraic geometry. II, volume 49 of Ergebnisse der Mathematik und ihrer Grenzgebiete. 3. Folge. A Series of Modern Surveys in Mathematics [Results in Mathematics and Related Areas. 3rd Series. A Series of Modern Surveys in Mathematics]. Springer-Verlag, Berlin, 2004. Positivity for vector bundles, and multiplier ideals. $\uparrow 20$

[Miy87] Yoichi Miyaoka. The Chern classes and Kodaira dimension of a minimal variety. In $\mathrm{Al}$ gebraic geometry, Sendai, 1985, volume 10 of Adv. Stud. Pure Math., pages 449-476. NorthHolland, Amsterdam, 1987. $\uparrow 2,14,15,23$

[MP97] Yoichi Miyaoka and Thomas Peternell. Geometry of higher-dimensional algebraic varieties, volume 26 of DMV Seminar. Birkhäuser Verlag, Basel, 1997. $\uparrow 14,15,16$

[Ros68] Hugo Rossi. Picard variety of an isolated singular point. Rice Univ. Studies, 54(4):63-73, 1968. $\uparrow 14$

[SBW94] Nicholas I. Shepherd-Barron and Pelham M.H. Wilson. Singular threefolds with numerically trivial first and second Chern classes. J. Algebraic Geom., 3(2):265-281, 1994. $\uparrow 3,21$

[Sim92] Carlos T. Simpson. Higgs bundles and local systems. Inst. Hautes Études Sci. Publ. Math., 75:5-95, 1992. $\uparrow 3,17,19,20$

DANIEL GREB, ESSENER SEMINAR FÜr Algebraische GeOMETRIE Und ARITHMETIK, FAKUltät FÜR MATHEMATIK, UNIVERSITÄT DUISBURG-ESSEN, 45117 ESSEN, GERMANY

E-mail address: daniel.greb@uni-due.de

URL: http://www . esaga.uni-due.de/daniel.greb/

Stefan Kebekus, Mathematisches Institut, Albert-Ludwigs-Universität Freiburg, ECKERSTRASSE 1, 79104 FREIBURG IM BREISGAU, GERMANY, AND UNIVERSITY OF STRASBOURG INSTITUTE FOR AdVANCED StUdy (USIAS), STRASBOURG, FRANCE

E-mail address: stefan.kebekus@math.uni-freiburg. de

URL: http://home.mathematik.uni-freiburg.de/kebekus

Thomas Peternell, Mathematisches Institut, Universität Bayreuth, 95440 BAyreuth, GERMANY

E-mail address: thomas . peternell@uni-bayreuth.de

URL: http://btm8x5.mat.uni-bayreuth.de/mathe1 